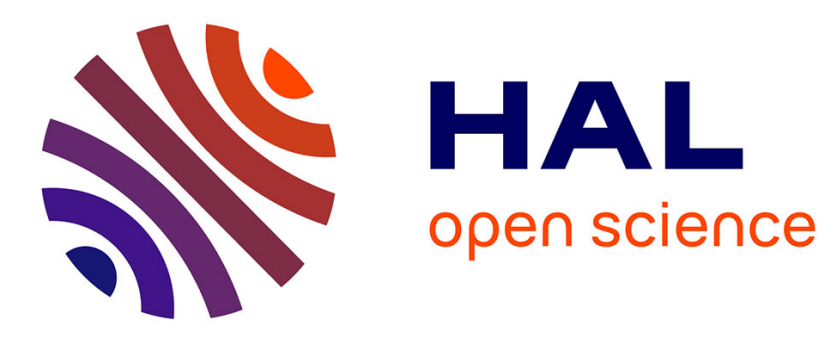

\title{
Slow dynamics in glass-forming materials
}

Frederic Affouard, Patrice Bordat, Adrien Lerbret, Marc Descamps

\section{To cite this version:}

Frederic Affouard, Patrice Bordat, Adrien Lerbret, Marc Descamps. Slow dynamics in glass-forming materials. Molecular Simulation, 2007, 32 (12-13), pp.1057-1068. 10.1080/08927020600900329 . hal00514999

\section{HAL Id: hal-00514999 \\ https://hal.science/hal-00514999}

Submitted on 4 Sep 2010

HAL is a multi-disciplinary open access archive for the deposit and dissemination of scientific research documents, whether they are published or not. The documents may come from teaching and research institutions in France or abroad, or from public or private research centers.
L'archive ouverte pluridisciplinaire HAL, est destinée au dépôt et à la diffusion de documents scientifiques de niveau recherche, publiés ou non, émanant des établissements d'enseignement et de recherche français ou étrangers, des laboratoires publics ou privés. 


\section{Molecular Simulation}

Journal of

Experimental Nanoscience

- Taylor \& Francis

Taylor \& Francis Group

\section{Slow dynamics in glass-forming materials}

\begin{tabular}{|r|l|}
\hline Journal: & Molecular Simulation/Journal of Experimental Nanoscience \\
\hline Manuscript ID: & GMOS-2006-0078.R1 \\
\hline Journal: & Molecular Simulation \\
\hline Date Submitted by the \\
Author: & 20-Jun-2006 \\
\hline Kemplete List of Authors: & $\begin{array}{l}\text { Affouard, frederic; LDSMM, Universite LIlle 1 - UMR CNRS } 8024 \\
\text { Bordat, Patrice } \\
\text { lerbret, adrien; Cornell University } \\
\text { descamps, marc; LDSMM }\end{array}$ \\
\hline Keywords: & $\begin{array}{l}\text { orientational disordered crystals, glassforming liquids, fragility, } \\
\text { liquid-glass transition, Mode-Coupling Theory }\end{array}$ \\
\hline
\end{tabular}

SCHOLARONE ${ }^{m}$

Manuscripts 


\title{
Slow dynamics in glass-forming materials
}

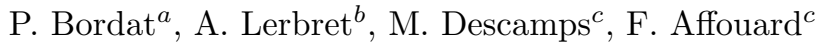 \\ ${ }^{a}$ Laboratoire de Chimie Théorique et de Physico-Chimie Moléculaire \\ UMR 5624, Université de Pau et des Pays de l'Adour, 64000 Pau, France \\ ${ }^{b}$ Department of Food Science, Cornell University \\ 103 Stocking Hall, Ithaca, New York, 14853 \\ ${ }^{c}$ Laboratoire de Dynamique et Structure des Matériaux Moléculaires, \\ CNRS UMR 8024, Université Lille I, \\ 59655 Villeneuve d'Ascq Cedex France \\ (Received 00 Month 200x; In final form 00 Month 200x)
}

\begin{abstract}
Dynamical properties of glass-formers such as glassy crystals, molecular liquids and model atomic liquids have been investigated in

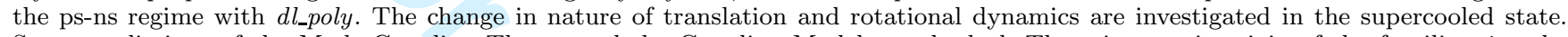
Some predictions of the Mode-Coupling Theory and the Coupling Model are checked. The microscopic origin of the fragility, i.e. the characteristic parameter involved in the liquid-glass transition, is also highlighted: the interaction potential, especially its anharmonicity and capacity for intermolecular coupling, is the key parameter controlling both the long time dynamics in supercooled systems and the short time dynamics in their glassy states.
\end{abstract}

Keywords: orientational disordered crystals; glassforming liquids; fragility; liquid-glass transition; Mode-Coupling Theory; Coupling Model; anharmonicity.

\section{Introduction}

It is well known that very different materials such as: silica, low-molecular-weight liquids (carbohydrates, alcohols), polymers or even proteins are able to exhibit a very intriguing feature called glass transition (1). This latter is characterized by an extraordinary decrease of several orders of magnitude of the mobility in a narrow temperature range without significant structural changes.

Understanding of the mechanisms leading to the glass transition has been a subject of intense research in recent years and it is still a matter of numerous theoretical and experimental studies (1).

Much attention has been recently devoted to the high temperature pico-nanosecond regime (ps-ns) of liquid glass-formers. A fundamental question concerns the microscopic description of the cooperative mechanisms which develop over a temperature range in which dynamics start being both relatively well described by the predictions of the mode coupling theory (MCT) and influenced by the potential energy landscape $(2 ; 3)$.

So far, MCT (4) is the only theory which provides a microscopic description of supercooled atomic liquids. The intrinsic basis of MCT states that the behavior of any time-dependent correlators describing the dynamics of the system is only controlled by its static density correlator $S(Q)$ and predicts the existence of a critical temperature $T_{c}$ corresponding to an ergodic to non-ergodic transition. Scaling properties predicted by MCT approaching $T_{c}$ have been successfully validated in numerous experiments $(5 ; 6 ; 7 ; 8)$ and molecular dynamics (MD) simulations (9).

It is now well accepted that $T_{c}$ also marks the crossover to the "landscape dominated" regime, as advocated long time ago by Goldstein (10), where dynamics can be described as thermally activated hoppings between inherent structures (minima of the potential energy). It has been demonstrated that an other remarkable crossover temperature $T_{A}$ can be identified above $T_{c}$ which coincides with the onset of non-exponential relaxation i.e the "landscape influenced" regime $(11 ; 3)$.

The aim of this paper is to present and discuss results obtained from MD simulations on slow dynamics

Molecular Simulation

ISSN 0892-7022 print/ISSN 1029-0435 online (c) 200x Taylor \& Francis

http://www.tandf.co.uk/journals

DOI: $10.1080 / 0892702 Y Y x x x x x x x x$

http://mc.manuscriptcentral.com/tandf/jenmol 
of glass-forming systems involving different degrees of freedom (translation and rotational): glassy crys-

tals, glycerol solutions and binary mixtures of Lennard-Jones (LJ) atoms. Dynamical properties of thesPage 2 of 30 compounds have been particularly investigated in the ps-ns regime where a change in the nature of the dynamics (fast to slow dynamics) are expected to emerge in the $\left[T_{A}-T_{c}\right]$ temperature range.

\section{Two-step relaxation and Mode Coupling Theory}

The idealized version (4) of this theory predicts a two-step relaxation scenario (fast $\beta$, slow $\alpha$ ) of all the time dependent correlators $\phi(q, t)$. MCT particularly states the following points: At short time, $\phi(q, t)$ decays to a plateau value, the so-called nonergodicity parameter classically noted $f_{q}^{c}$. The dynamical regime associated with this plateau is called $\beta$. It is centered around the rescaling time $t_{\sigma}$ which is given by $t_{\sigma}=t_{0}|\sigma|^{-1 / 2 a}$ where $t_{0}$ is a characteristic microscopic time and $\sigma$ is proportional to $\left|T-T_{c}\right|$. Above $T_{c}$, the late $\beta$ regime or the early $\alpha$ relaxation is described by the following power law (going beyond first order):

$$
\phi(q, t)=f_{q}^{c}-h_{q}^{(1)} \cdot(t / \tau)^{b}+h_{q}^{(2)} \cdot(t / \tau)^{2 b}
$$

where the first two terms correspond to the classical von Schweidler law, the last term is a second order correction and $\tau=t_{0}|\sigma|^{-\gamma}$ with $\gamma=1 / 2 a+1 / 2 b$. At long time, MCT also predicts that the previous fast regime is followed by a slow relaxation, called $\alpha$, with the characteristic time $\tau$. Furthermore, parameters $a$ and $b$ are temperature and $q$ independent and related via $\Gamma^{2}(1-a) / \Gamma(1-2 a)=\Gamma^{2}(1+b) / \Gamma(1+2 b)$ where $\Gamma(x)$ is the gamma function.

\section{Glassy Crystals}

Most of the glassformers are molecular liquids and accordingly possess both translational and rotational degrees of freedom (TDOF and ODOF). Approaching the glass transition, the coupling between TDOF and ODOF is far from being understood and the precise role of the ODOF remains unclear as demonstrated by the so-called low-temperature translation-rotation paradox $(12 ; 13)$. It is now well established that the phenomenology of glass formation is also displayed by some partially disordered molecular crystals i.e. plastic crystals in which the average position of the centers of mass are ordered on a lattice while the orientations are dynamically disordered $(14 ; 15)$. Indeed, some of them, called glassy crystals (16), such as cyanoadamantane $(17)$, ethanol $(18 ; 19)$, cyclooctanol $(20)$ or difluorotetrachloroethane $(21)$ can be deeply supercooled and present many properties characteristic of the conventional molecular liquid glasses such as a step in the specific heat at the glass transition or a non-Arrhenius behavior of the relaxation times. Those compounds has led recently to a strong interest since they offer valuable possibilities to focus mainly on the role of the orientational degrees of freedom (ODOF) whose the importance during the glass formation remains a matter of debate at present. It has to be noticed that glassy crystals in opposition to the so called "orientational glasses" like mixed crystals $\operatorname{Ar}_{1-\mathrm{x}}\left(\mathrm{N}_{2}\right)_{\mathrm{x}}$ or $(\mathrm{KCN})_{\mathrm{x}}(\mathrm{KBr})_{1-\mathrm{x}}$ are not frustrated by quenched disorder externally imposed by a dilution (22).

MCT has been initially developed to provide a microscopic understanding of simple atomic liquids. However, general predictions have also been successfully validated for molecular compounds. Recently, some extensions of MCT called molecular mode coupling theory (MMCT) (9) have been proposed to take ODOF into account: one diatomic probe molecule in an atomic liquid (23), liquids made of linear molecules (24) or water (25) to cite only a few. The authors have particularly shown that some of the basic predictions of MCT still hold owing TDOF/ODOF coupling. As revealed by recent MD investigations (26; 27) performed on orthoterphenyl (OTP), coupling of the rotational dynamics to the center-of-mass motion can be complex. No microscopic theory has been developed for plastic crystals so far. However, in (28; 29; 30), we have particularly shown from NMR and Raman experiments, and MD computer simulations that some predictions of the idealized version of the MCT (critical temperature $T_{c}$ and time scaling laws) 
Page 3 of 30 tic crystals.

In the following, we investigated three models of plastic crystals possessing different kind of crystalline and molecular symmetries and different type of reorientational dynamics. The present numerical simulation investigations aim to demonstrate that plastic crystals whose the dynamics are almost completely controlled by rotation shares some common dynamical features with liquid glass-formers in the ps-ns regime where some precursor features (non-exponentiality or non-Arrhenius behavior) of the glass transition emerge.

\subsection{Models}

3.1.1 Chloroadamantane. Chloroadamantane $\mathrm{C}_{10} \mathrm{H}_{15} \mathrm{Cl}$ (CLADM) is a rather huge molecule which belongs to the substituted adamantane family. It shows a plastic phase structure isomorphous to cyanoadamantane, but the chloroadamantane molecule possesses a smaller substitute and a faster dynamics well adapted for MD simulation investigations. Rotational motions are also suspected to change in nature as it is reported from earlier incoherent quasielastic neutron scattering experiments (31). Chloroadamantane undergoes at $244 \mathrm{~K}$ a first order transition from an ordered monoclinic structure to a rotator phase with face-centered-cubic (fcc) symmetry (32). The plastic-liquid transition occurs at $442 \mathrm{~K}(32$ ).

The simulated system is composed of rigid linear molecules with two sites: one chlorine atom (noted $\mathrm{Cl}$ ) and one super atom (noted $\mathrm{Adm}$ ) that models the adamantane part $\mathrm{C}_{10} \mathrm{H}_{15}$. The distance of both pointlike atoms is $3.3 \AA$ and the molecule's moment of inertia is $302.733 \mathrm{amu} . \AA^{2}$. Rigid body equations with quaternions were used for the the orientational variables.Molecular dynamics calculations were performed on a system of $N=256$ molecules $(4 \times 4 \times 4$ fcc crystalline cells) interacting through a Lennard-Jones short range site-site potential of the form

$$
v(r)=4 \epsilon\left((\sigma / r)^{p}-(\sigma / r)^{q}\right)
$$

where $r$ is the distance between two different sites. The parameters $\epsilon, \sigma, p$ and $q$ are specified in table 1 .

A complete description of a similar model used previously to simulate cyanoadamantane using the Monte Carlo technique is given in $(33 ; 34)$, so we give here only the essential details. The parameters of the $\mathrm{Cl}-\mathrm{Cl}, \mathrm{C}-\mathrm{C}$ and $\mathrm{H}-\mathrm{H}$ potentials were taken from potential energy calculations of chlorine atoms in molecular crystals (35). The parameters for Adm interactions have been determined from the atom-atom interactions between atomic sites of the adamantane part of the molecules (36). First, the averaged energies over all molecular orientations were calculated for different distances of centers of mass of the adamantane part of molecules. Then, they were fitted into the Lennard-Jones form (seei table 1). The parameters for interactions between dissimilar sites have been determined by the Berthelot combining rules. The 12-6 Lennard-Jones functions were applied for all dissimilar interactions.

The chloroadamantane molecule possesses a relatively large dipolar moment $\vec{\mu}$ (2.39 Debyes) which is parallel to the molecular axis. The electrostatic interactions were handled by the Ewald method with two partial charges $(q= \pm 0.151 e)$ localized on both sites. This simple charge distribution has been constructed to make the total molecular charge equal to zero and to reproduce the total dipole moment. Newton's equations of motion were solved with a time step of $\Delta t=5 \mathrm{fs}$. We worked in the NPT (constant number of molecules, temperature and pressure) statistical ensemble using the Melchionna modification of the Hoover algorithm with a thermostat and barostat relaxation time of 1 ps (37). Periodic boundaries conditions were used. MD simulations have been performed at 29 different temperatures from $T=220$ to $500 \mathrm{~K}$ for a sample corresponding to the crystalline fcc rotator phase.

It should be noted that the CLADM model is particularly oversimplified. The main reason of this simplification is due to the large dipole moment of the CLADM molecule which requires the calculation of the electrostatic interactions unlikely to the Norbornylene or the difluorotetrachloroethane models (see below). However, it has been precisely constructed to keep the main experimental features and to speed up simulations in order to reach the nanosecond regime. Several comparisons of the results obtained from the CLADM model with experimental data have been published in (28). In this study, from calculations 
of the enthalpy, it was shown that this model captures the anomaly found in calorimetric experiments by Oguni et Al. (38) close to the Arrhenius to Non-Arrhenius deviation reported in the present manuscriptPage 4 of 30 Moreover, both high- and low-temperature apparent activation energies of the relaxational time as defined in the present manuscript were found to be in good agreement with NMR experimental results. In (39), it is also reported that the shrinking of the lattice constant found in good agreement with X-ray experiments leads to an increase of the steric hindrance with decreasing temperature and in turn to a slowing down of the orientational dynamics.

3.1.2 Norbornylene. Norbornylene (NBE) $\mathrm{C}_{7} \mathrm{H}_{10}$ displays one plastically crystalline over the temperature range $129 \mathrm{~K}$ to $320 \mathrm{~K}$ (40). The plastic crystal structure is hexagonal close-packed (hcp) (41). The structure of the brittle phase is reported to be monoclinic $P 2_{1} / c$ from ab-initio calculation (42). At high temperature, an isotropic reorientational motion is found (43) whose relaxational time follows an Arrhenius law. No discontinuity for this process is found at the plastic to liquid transition. Below $160 \mathrm{~K}$, an abrupt dynamical change is mentioned with anisotropy setting.

The simulated sample consists of $N=686$ molecules located on an average hcp lattice corresponding to a sample of $7 \times 7 \times 7$ unit cells. Each molecules $\mathrm{C}_{7} \mathrm{H}_{10}$ is described by its 17 atoms and considered as rigid.Rigid body equations with quaternions were used for the the orientational variables.Due to the presence of a carbon-carbon double bond, NBE possesses a small dipolar moment which was determined to be $0.32 \mathrm{D}$. As already made by Min et al. (42) in their ab initio determination of the NBE low temperature crystalline structure, the electrostatic contributions have been neglected in our simulation. Therefore, we have just considered a Buckingham atom-atom potential whose coefficients are given in table 2. Despite this approximation, both structure and dynamics were found in good agreement with experimental results. Newton's equations of motion were solved with a time step of $\Delta t=5 \mathrm{fs}$. We worked in the NPT statistical ensemble with periodic boundary conditions at constant atmospheric pressure. We used Berendsen barostat and thermostat with relaxation times of $2.0 \mathrm{ps}$ and $0.2 \mathrm{ps}$ for controling the pressure and the temperature respectively (44). MD simulations have been carried out at 28 different temperatures from $T=125$ to 320 $\mathrm{K}$ for a sample corresponding to the experimental crystalline hcp rotator phase. Below $125 \mathrm{~K}$, the system falls out of equilibrium over the MD time window and becomes non ergodic.

3.1.3 Difluorotetrachloroethane. Difluorotetrachloroethane (DFTCE) is composed of simple molecules $\mathrm{CFCl}_{2}-\mathrm{CFCl}_{2}$ close to dumbbells extensively used in $\mathrm{MD}$ calculations as prototype of molecular glassformers liquids $(45 ; 46 ; 47)$. DFTCE has been experimentally widely studied and presents a rich variety of unusual properties. DFTCE exhibits a glass transition of the overall rotation of the molecules at $T_{g}=$ $86 \mathrm{~K}(48)$. Two additional heat anomalies are found at 60 and $130 \mathrm{~K}$ and associated respectively to a sub- $T_{g} \beta$ process and the freezing of the transformation between trans and gauche conformation of the molecule. Changes concerning the nature of dynamics in this system has been reported from NMR experiments (49), Brillouin and dielectric spectroscopy (21). In this latter investigation, a crossover from individual to correlated rotational motions was particularly suggested.

MD calculations were performed on a system of $N=686(7 \times 7 \times 7$ bcc crystalline cells $)$ molecules. Each DFTCE molecule is described by its 8 atoms and considered as a rigid unit. Rigid body equations with quaternions were used for the the orientational variables. They interact through a Buckingham short range atom-atom potential (see parameters in table 3) and the electrostatic contributions have been neglected since the DFTCE molecule possesses a weak dipolar moment. No structural or dynamical change has been found for a system where electrostatic interactions are taken into account. Newton's equations of motion were solved with a time step of $\Delta t=5 \mathrm{fs}$. We worked in the NPT statistical ensemble with periodic boundary conditions where the simulation box is allowed to change in size and shape using the Melchionna modification of the Hoover algorithm (37) with a thermostat and barostat relaxation time of 1 ps. MD simulations were done for a sample corresponding to the DFTCE orientationally disordered phase at 14 different temperatures from $T=130$ to $260 \mathrm{~K}$ in steps of $10 \mathrm{~K}$. It should be mentioned that our very simple DFTCE model allows us to perform very long MD runs of about 50 ns. 


\section{$3.2 \quad$ Results and Discussion}

Single-molecule reorientational dynamics can be investigated from the self angular correlation functions $C_{l=1,2}$ defined as:

$$
C_{l}(t)=\frac{1}{N} \sum_{i=1, N}\left\langle P_{l}\left(\vec{u}_{i}(t) \cdot \vec{u}_{i}(0)\right)\right\rangle
$$

where $P_{l}$ is the $l$-order Legendre polynomial and $\vec{u}_{i}$ a unit vector parallel to the dipole moment of each molecule $i$ in the case of CLADM and NBE or directed along the $\mathrm{C}_{3}$-symmetry carbon-carbon molecular axis of molecule $i$ in the case of the DFTCE molecules. From $C_{l=1,2}$, we can obtain clarification on the nature of dynamical changes and anisotropy of the molecular motions. Experimentally, $C_{1}(t)$ can be directly measured in dielectric relaxation and $C_{2}(t)$ in Raman scattering (14). $C_{l=1,2}$ can also be related to the informations obtained from NMR relaxation measurements.

Figure 1 shows the $C_{2}(t)$ time correlation functions for all models and investigated temperatures. Clearly, at least for the lowest temperatures, a two step relaxation behavior as predicted by MCT and already observed in supercooled molecular liquids $(45 ; 50)$ is shown in our simulations. At very short times, we observe a fast decay corresponding to a microscopic regime which is modulated by vibrational motions. This latter behavior is followed by a plateau-like region, which becomes more pronounced at the lowest temperatures. This feature is identified with the $\beta$ regime. At the end, the last step is a decay to zero corresponding to the $\alpha$ process. $C_{1}(t)$ time correlation functions, not displayed in the present paper, show a very similar behavior to the $C_{2}(t)$ functions. When lowering the temperature, an intermediate plateau region emerges which proves the presence of an orientational caging between neighboring molecules (see Fig. 2).

This is the rotational analogue of the translational cage effect observed in liquids (see Ref. (51)). This transient regime is followed by the $\alpha$ process which can be associated in plastic crystals with large tumbling motion between preferred molecular orientations as displayed in Fig. 2 for CLADM. A consequence is that the tumbling of one molecule is allowed only if an orientational rearrangement of its local neighbors occurs. This cooperative motion is clearly displayed using projections of the individual dipolar moments on one crystallographic plane.

The $\tau_{1}$ and $\tau_{2}$ relaxational times are defined as the time it takes for their respective time correlation functions to decay $e^{-1}$ of their initial values. At high temperature, for all correlators, $\tau(T)$ can be well fitted with an Arrhenius law $\tau(T)=\tau_{0} \exp \left(E_{0} / T\right)$ where the parameters $\left(\tau_{0}, E_{0}\right)$ allow us to calculate the temperature dependent activation energy $E(T)=T \cdot \log \left(\tau / \tau_{0}\right)$.

Figure 3 displays $E(T) / E_{0}$ as function of the temperature. Clearly, at a remarkable temperature identified to $T_{A}$, all activation energies start diverging simultaneously from unity corresponding to the Arrhenian high temperature behavior. This is an indication of the existence of a dynamical crossover from a free diffusion regime to the so-called landscape-influenced regime occurring at $T_{A}$ corresponding to a transition from quasi-free rotational diffusion of the molecular dipoles to activated geared tumblings as seen in Fig. 2.

The nature of the rotational motions involved in this dynamical change is given by the ratio $\tau_{1} / \tau_{2}$ in Fig. 4.

Upon lowering the temperature, we observe that $\tau_{1} / \tau_{2}$ clearly deviates from 3 and approaches 1 . For uncorrelated small angular steps motion, it can be shown that the orientation correlation functions follow an exponential decay $C_{l}=\exp \left[-l(l+1) D_{r} t\right]$ where $D_{r}$ is the rotational diffusion coefficient. $\tau_{1} / \tau_{2}=3$ is therefore expected for free small-step rotation diffusion type of motion while $\tau_{1} / \tau_{2}=1$ is classically associated to activated jump-like motion (52). These results prove the existence of a plastic-plastic transition which can be interpreted as the rotational analogue of the Goldstein crossing temperature seen in liquids.

\section{Glycerol molecular liquid}

We now focus on the dynamical properties of molecular liquids which present a slowing down upon cooling. Among them, we have studied glycerol for its key role in bioprotection. Indeed, it is well known that 
disaccharide/water binary solutions are able to protect biomolecules against dessication, dehydration and this ability seems to be strengthened by diluting a small amount of glycerol in these sugar/water solutionsPage $\mathbf{6}$ of $\mathbf{3 0}$ However, the microscopic mechanism responsible for the biopreservation are still unclear and several hypotheses non fully satisfactory have been proposed $(53 ; 54 ; 55 ; 56)$. We have realized a comparative study of the disaccharide/water solutions $(57 ; 58)$ by looking at both the structure and the dynamics in order to extract characteristic parameters playing a key role in the bioprotection mechanisms. The next step is the study of ternary mixtures first implying the development of a good model of the glycerol liquid to reproduce the main dynamical features observed experimentally and presented below.

\subsection{Computational details}

The system is composed of 108 flexible glycerol molecules modeled using the all-atom AMBER force field (59) employed by Chelli et al. (60). This model produces a structure in good agreement with the experimental one but the dynamical properties can be improved. Then, the atomic charges and the LennardJones parameters of the hydroxyl groups have been reparametrized (61) in order to reproduce more realistically the diffusion coefficient $D(62)$ and the characteristic times $\tau_{Q}$ obtained from Neutron Spin Echo experiments (63). Electrostatic interactions were handled by the reaction-field method with $\varepsilon_{R F}=40$ close to the values found in the literature (64). The reaction field method has been compared to the Ewald summation on glycerol liquid and neither structural nor dynamical changes have been noticed. As glycerol molecules are entirely flexible with slow and fast internal motions (vibrations), the time step is as small as $0.5 \mathrm{fs}$ (typically one twentieth of the highest frequencies of the vibrational modes). The cutoff radius is 10 $\mathrm{A}$ and cubic periodic boundary conditions have been applied. Molecular Dynamics simulations have been performed in the NPT statistical ensemble (44) at constant standard pressure (1 bar) using a barostat relaxation time of $1 \mathrm{ps}$ and a thermostat relaxation time of $0.2 \mathrm{ps}$. The temperatures range from $313 \mathrm{~K}$ to $493 \mathrm{~K}$ in steps of $20 \mathrm{~K}$ and the simulation lengths vary from 0.7 to $6 \mathrm{~ns}$.

\subsection{Results and Discussion}

The intermediate scattering function $S(\vec{Q}, t)$ usually obtained from coherent neutron scattering experiments has been analyzed in order to investigate numerically collective dynamics.

The static structure factor $S(Q)$ defined as $S(Q, t=0)$ is displayed in Fig. 5(a). A qualitative agreement with experimental data extracted from Ref. (65) is observed. This indicates that both real and model systems exhibit similar ordering. An interesting new point is the existence of a pre-peak $(61)$ close to $\mathrm{Q} \approx$ $0.6 \mathrm{~A}^{-1}$ located below the first sharp diffraction peak at $\mathrm{Q}=\mathrm{Q}_{\max } \approx 1.4 \mathrm{~A}^{-1}$. This pre-peak seems to be also present in experimental data (65) at lower temperature. This feature has already been observed in some other hydrogen-bonded glass-forming molecular liquids such as metatoluidine and metacresol (66; 67). In such liquids, the pre-peak has been interpreted as a signature of structural heterogeneities corresponding to clusters of hydrogen-bonded molecules and forming a medium range order.

The dynamical structure factor $S(Q, t)$ has been calculated from MD simulations at different temperatures and different $Q$ wave-vectors (61) and is compared with the Neutron Spin Echo data (63) in Fig. 6 at $\mathrm{Q}=\mathrm{Q}_{\max }$. Despite slight discrepancies at the highest and the lowest temperatures, a reasonable agreement between experimental and numerical $S(Q, t)$ is found. Upon cooling, $S(Q, t)$ displays a decay usually observed in MD simulations (27) as well as in experiments on glass-forming molecular liquids (63; 68). The shape of $S(Q, t)$ is similar to that drawn for glassy crystals in section 3 . It is analyzed in terms of $\alpha$ - $\beta$ relaxations in the framework of $\mathrm{MCT}(4)$. In the $\alpha$ long time relaxation regime, the characteristic time $\tau_{Q}$ is defined as the time it takes for $S(Q, t)$ to decay from 1 to $1 / e$. The characteristic time $\tau_{Q_{m a x}}$ and the diffusion coefficient $D$ obtained experimentally and numerically are shown in Fig. 7 .

The characteristic time $\tau_{Q_{\max }}$ and the diffusion coefficient $D$ are now consistent with previous experimental results $(63 ; 62)$ and exhibit a non-Arrhenius dynamical behavior. According to MCT, any characteristic relaxation times should yield asymptotically the same temperature dependence $\approx\left(T-T_{c}\right)^{-\gamma}$. But an accurate determination of $T_{c}$ is difficult due to the limited temperature range investigated in Ref. (61). We can just check that the values $\left(T_{c} \approx 288 \mathrm{~K}\right.$ and $\left.\gamma \approx 2.87\right)$ found in Ref. (69) are compatible with the curves 
displayed in Fig. 7.

Page 7 of 3 nother intrinsic basis of MCT states that the behavior of any time-dependent correlators such as $S(Q, t)$ describing the dynamics of the system is only controlled by its static density correlator $S(Q)$. This statement has been nicely demonstrated both experimentally and numerically for the pure van der Waals glass-forming ortho-therphenyl system $(70 ; 27)$. Fig. $5(\mathrm{~b})$ shows the $Q$-dependence of the relaxation time $\tau_{Q}$, which is modulated in phase with $S(Q)$. Moreover, the curve $\tau_{Q}(Q)$ highlights the pre-peak $($ at $\approx 0.6$ $\mathrm{A}^{-1}$ ) and provides an estimation of the lifetime of the hydrogen-bonded molecules clusters, $\tau \approx 80$ ps.

\section{Binary mixtures of Lennard-Jones atoms}

From sections 3 and 4, we have seen that different molecular systems could exhibit similar dynamical behaviors upon cooling. However, the different kinds of interactions and the different sizes of the molecules make the comparisons ambiguous. Therefore, we have chosen to study the influence of the shape of the interaction potential on the dynamical properties of binary mixtures of LJ atoms.

\subsection{Model}

MD simulations have been carried out on binary particles mixtures with three different interaction potential (see Fig. 8). We have performed from $10^{5}$ to $6 \times 10^{7}$ time steps, depending on temperature. All models are composed of 1500 uncharged particles (1200 of species A and 300 of species B). Particles interact via the $(q, p)$ Mie potential having the form:

$$
V(r)=\frac{E_{0}}{(q-p)}\left(p\left(\frac{r_{0}}{r}\right)^{q}-q\left(\frac{r_{0}}{r}\right)^{p}\right)
$$

The parameters $r_{0}$ and $E_{0}$ represent the position of the minimum of the well and its depth, respectively. The reduced LJ units (71) are used. The choice of $q=12$ and $p=6$ corresponds to the standard LJ potential (see Table 4) used by Kob \& Andersen (K\&A), which has been studied extensively as a model glassformer $(72 ; 73 ; 74 ; 75)$.

For the purpose of investigating the change of dynamics with controlled change of $V(r)$, we developed two other models by changing only the exponents, $q$ and $p$, of the Mie potential for the A-A interaction. They are $(q=8, p=5)$ and $(q=12, p=11)$ and shown together with the $(12,6)$ Lennard-Jones potential in Fig. 8. The $(12,11),(12,6)$ and $(8,5)$ potentials are referred to as Model I, II and III, respectively. The well depth and the position of the minimum of $V(r)$ are unchanged and the standard $(12,6)$ Lennard-Jones potentials of the K\&A model for the A-B and the B-B interactions are used in order to retain as much as possible the remarkable ability of the K\&A model to form a glass upon cooling. The $(12,11)$ model is more harmonic than the classical $(12,6)$ potential, while the $(8,5)$ potential is a flat well and exceedingly anharmonic.

\subsection{Role of the shape of the interacting potential on the long time dynamics}

From the self intermediate scattering function $F_{S}\left(Q_{0}, t\right)$ at $Q_{0}=2 \pi / r_{0}$ where $r_{0}$ is the position of the maximum of the first peak of the radial distribution function, we have determined a long relaxation time $\tau$ corresponding to the $\alpha$ process, when $F_{S}\left(Q_{0}, t\right)$ has decayed by a factor of $e^{-1}$ from the plateau-like region. In the MCT framework (see section 2), $\tau$ is proportional to $\left(T-T_{C}\right)^{-\gamma}$. In order to check the validity of this scaling law, we have first determined the exponent $b$ of Eq. 1 from the master-curve of $F_{S}\left(Q_{0}, t\right)$ as displayed in Fig. 10. Another method would consist in using the factorization theorem in the $\beta$ regime as described in (30). Knowing $b$, we have deduced $a$, leading to a value for the exponent $\gamma$. Once $\gamma$ is known, we have fitted the $\alpha$ relaxation times $\tau(T)^{-1 / \gamma}$ by a linear law of the form $\left(T-T_{C}\right)$ in order to get the critical temperature $T_{C}$ of MCT. This linear behavior, only valid close to $T_{C}$, is shown in Fig. 9 for the three studied systems. 
From the self intermediate scattering functions $F_{S}\left(Q_{0}, t\right)$ and $\tau_{A}$ (the $\alpha$ relaxation time $\tau$ of species $\left.\mathrm{A}\right)$ at different temperatures for the three models, we can plot a master-curve obtained from the superposition oPage $\mathbf{8}$ of $\mathbf{3 0}$ $F_{S}\left(Q_{0}, t / \tau_{A}\right)$ in the long time regime. The master-curve of Model III is displayed in Fig. 10. Upon lowering the temperature from the normal liquid state, we observe that the self intermediate scattering functions progressively collapse onto an apparent master curve. However, a detailed view of this thick curve, shown in the inset of Fig. 10, reveals that the different functions do not strictly superpose. Therefore, it is only approximatively possible to extract a temperature independent stretched exponent $\beta$ using a KohlrauschWilliams-Watt (KWW) fit of the master curve in a restricted temperature range. The same also stands for Models I and II. We have obtained $\beta=0.84,0.81$ and 0.76 for Models I, II and III respectively. The error bar is about 0.1 due to the thickness of the master-curve.

\subsection{Beyond MCT analysis, the binary LJ liquid-glass transition}

5.3.1 Anharmonicity and intermolecular coupling. Since a strict superposition of $F_{S}\left(Q_{0}, t / \tau_{A}\right)$ functions does not hold, it is interesting to consider a temperature dependent stretched exponent $\beta$ which is displayed in Fig. 11.

$\beta$ is observed to continuously decrease from 1 at high temperatures to $0.69,0.65$ and 0.60 at very low temperatures below $T_{C}$ for Models I, II and III respectively. Moreover, $\beta$ obtained from Model I is always found higher than $\beta$ of Model II, itself higher than $\beta$ of Model III. Therefore, the stretched exponent $\beta$ decreases as anharmonicity increases. This behavior indicates that the anharmonicity of the intermolecular potential significantly controls the long range interactions between particles and therefore their collective relaxations. This result strongly supports the idea developed in the Coupling Model (76) that the intermolecular coupling measured by the parameter $n=(1-\beta)$ controls relaxations in liquids.

5.3.2 Link between short time and long time dynamics. We can focus now on the liquid-glass transition by looking at a characteristic parameter, that is the fragility $m$ (defined below, see Eq. 5), and its sensitivity to the shape of the potential. The $\alpha$ relaxation time, $\tau$, of all glass-forming liquids increases on cooling and becomes so long at some temperature $T_{g}$ that equilibrium cannot be maintained and the liquid is transformed to a glass as $\tau$ reaches commonly $10^{2} \mathrm{~s}$. This behavior is shared by very different glass-formers, but the temperature dependence of $\tau$ can differ greatly from one liquid to another $(77 ; 78)$. Therefore, the change of $\log (\tau)$ with $T_{g} / T$ at $T_{g} / T=1$, which is given by the steepness index or the fragility $m$, is defined by $(79 ; 80 ; 81)$

$$
m=\left.\frac{d \log (\tau)}{d\left(T_{g} / T\right)}\right|_{T_{g} / T=1} .
$$

The values of $m$ vary over a large range, from about 17 for strong glass-formers (like silica) to values as high as about 200 found for some glass-formers called fragile. Nevertheless, the microscopic origin of the large variation of the fragility $m$ is not clearly understood yet. Several attempts have been made (see Ref. (82) for a list and references therein), one being the correlation between the fragility $m$ and the vibrational properties of the glass at temperatures well below $T_{g}$ found recently by $\mathrm{T}$. Scopigno et al. (83). The nonergodicity parameter, $f(Q, T)$ at $T<<T_{g}$ is determined by vibrations and from inelastic X-ray scattering data, the temperature dependence of $f_{0}(T)=f(Q \rightarrow 0, T)$ is well described by the following relation:

$$
f^{-1}(Q \rightarrow 0, T)=1+\alpha \frac{T}{T_{g}}
$$

T. Scopigno et al. showed that $m$ and $\alpha$ are proportional for many glass-formers.

Before investigating the glassy state, we deal with the supercooled liquid state. In Fig. $12, \log \left(\tau_{A}\right)$ is plotted against $T_{r e f} / T$ and the data of the three models show systematic change. It can be seen that the 
slope, fragility index $m\left(\tau_{A}\right) \equiv \frac{d \log \left(\tau_{A}\right)}{d\left(T_{r e f} / T\right)}$ as $\left(T_{r e f} / T\right) \rightarrow 1$, increases monotonically in the order of Models I,

Page 9 of 30 and III. The values of $m\left(\tau_{A}\right)$ determined from this latter relation are 15.07, 18.57 and 26.58 for Models I, II and III. As a comparison, Sastry's method (2) gives values of $m\left(\tau_{A}\right)$ equal to $0.195,0.241$ and 0.405 for Models I, II and III, respectively. So, $m\left(\tau_{A}\right)$ increases with anharmonicity. We have also determined a steepness or fragility index, $m\left(D_{A}\right) \equiv \frac{d \log \left(1 / D_{A}\right)}{d\left(T_{r e f} / T\right)}$ as $\left(T_{r e f} / T\right) \rightarrow 1$ from the diffusion coefficient, $D_{A}$, of species A calculated from the mean-square displacement $\left\langle u^{2}(t)\right\rangle$ at long times when it reaches the linear $t$ dependence. Again, $m\left(D_{A}\right)$ increases monotonically in the order of Models I, II and III, or with anharmonicity.

So, the fragility $m$ determined from different dynamical parameters and the intermolecular coupling $n=(1-\beta)$ increase with anharmonicity. We study now the very low temperature domain, well below $T_{r e f}$ in order to concentrate on the vibrational properties of the three models in which relaxation is absent.

Therefore, the nonergodicity parameter $f(Q, T)$ determined from $F(Q, t)$ is contributed entirely from vibrations. In all three models, the dependence of $f_{0}(T)^{-1}$ on $T / T_{\text {ref }}$ shown in Fig. 13 is approximately linear, suggesting the following fitting law:

$$
f_{0}(T)^{-1}=1+\alpha \frac{T}{T_{r e f}}
$$

just similar to Eq. 6 used to represent the dependence of $f_{0}(T)$ on $T / T_{g}$ of real glass-formers obtained by inelastic X-ray scattering (83). We see in Fig. 13 that the increase of $f_{0}(T)^{-1}$ with $T / T_{r e f}$ is fastest for model III and slowest for model I, which means the slope $\alpha$ is largest for model III and smallest for model I. The parameter $\alpha$ increases with anharmonicity of the potential (like $m\left(\tau_{A}\right), m\left(D_{A}\right), n=(1-\beta)$ ) and is even proportional to $m\left(\tau_{A}\right)$ (see the inset of Fig. 13) as shown in (83). At this point, we can state that the interacting potential is the origin of the correlation of $\alpha$ with $m\left(\tau_{A}\right)$ or $m\left(D_{A}\right)$, and $n=(1-\beta)$, suggesting the same holds for real glass-formers.

Fig. 14 summarizes the value of the fragility $m$ estimated from different definitions for the three models identified by the intermolecular coupling parameter $n=(1-\beta)$. Whatever the definition used to calculate the fragility $m$, Fig. 14 shows that $m$ of Model I is smaller than $m$ of Model II, itself smaller than $m$ of Model III, and that the increase of $m$ is in relation with the increase of $n$. Moreover, before concluding, we observe the same trend for the fragility $m$ determined by the relation presented in Eq. 8 .

$$
m=\left.\frac{d \log f}{d T}\right|_{T=T_{r e f}^{-}}
$$

It is then important to note that the parameter $n$, which is a measure of the intermolecular coupling due to the anharmonicity of the interaction potentials also controls the short time dynamics observed via the nonergodicity parameter $f$ below $T_{r e f}$. This point is detailed in Ref. $(82 ; 84)$. So, the shape of the interaction potential and particularly its anharmonicity is the key parameter, which controls the long time relaxations in supercooled liquids, the vibrations in glasses and which link them together.

\section{Conclusion}

Very different systems like orientationally disordered crystals, molecular and atomic glassforming liquids presented here exhibit similar dynamical behaviors on cooling. Other systems, not shown in the present manuscript, such as polymers or proteins exhibit the same behaviors. The main features discussed above are:

- the two step $(\alpha-\beta)$ relaxation which emerges at $T_{A}$,

- the existence of a critical temperature $T_{C}$ corresponding to an ergodic to non-ergodic transition,

- the fragility controlled by the anharmonicity (intermolecular coupling) of the interaction potential,

- the link between short time and long time dynamics. 
The different systems considered above have been chosen to show the very diverse possibilities of $d l_{-} p o l y$.

The atomistic simulations are a very powerful tool in complement to theories and experiments, and permpage $\mathbf{1 0}$ of $\mathbf{3 0}$ us to find the microscopic mechanism responsible for macroscopic behaviors. A future challenge is a better description of the glassy state of glass-formers, which needs very long simulations that $d l_{-} p o l y \_3$ could achieve.

\section{Acknowledgments}

The authors wish to acknowledge the use of the facilities of the IDRIS (Orsay, France) and the CRI (Villeneuve d'Ascq, France) where calculations were carried out. This work was supported by the INTERREG III (FEDER) program (Nord Pas de Calais/Kent). 
[1] P. G. Debenedetti and F. H. Stillinger, Nature 410, 259 (2001).

[2] S. Sastry, Nature 409, 164 (2001).

[3] T. B. Schroeder, S. Sastry, J. C. Dyre, and S. C. Glotzer, J. Chem. Phys. 112, 9834 (2000).

[4] W. Götze, in Liquids Freezing and the Glass Transition, J. P. Hansen, D. Levesque and J. Zinn-Justin, North-Holland, 1990.

[5] A. Tölle, H. Schober, J. Wuttke, and F. Fujara, Phys. Rev. E 59, 809 (1997).

[6] W. Steffen, A. Patkowski, H. Gläser, and E. W. Fischer, Phys. Rev. E 49, 2992 (1993).

[7] P. Lunkenheimer et al., Phys. Rev. Lett. 77, 318 (1996).

[8] G. Hinze, D. Brace, S. Gottke, and M. Fayer, Phys. Rev. Lett. 84, 2437 (2000).

[9] R. Schilling, Theories of the structural glass transition, in Collective Dynamics of Nonlinear and Disordered Systems, G. Radons, W. Just, and P. Haeussler, Springer, 2003.

[10] M. Goldstein, J. Chem. Phys. 51, 3728 (1969).

[11] S. Sastry, P. G. Debenedetti, and F. H. Stillinger, Nature 393, 554 (1998).

[12] G. Heuberger and H. Sillescu, J. Phys. Chem. 100, 15255 (1996).

[13] F. R. Blackburn, C.-Y. Wang, and M. Ediger, J. Phys. Chem. 100, 18249 (1996).

[14] J. N. Sherwood, The Plastically Crystalline State, J. Wiley \& Sons, 1979.

[15] N. G. Parsonnage and L. A. K. Staveley, Disorder in Crystals, Clarendon, Oxford, UK, 1978.

[16] H. Suga and S. Seki, J. of Non-Cryst. Solids 16, 171 (1974).

[17] F. Affouard, J.-F. Willart, and M. Descamps, J. of Non-Cryst. Solids 307-310, 9 (2002).

[18] A. Criado et al., Phys. Rev. B 61, 12082 (2000).

[19] S. Benkhof, A. Kudlik, T. Blochowicz, and E. Rössler, J. Phys.: Condens. Matter 10, 8155 (1998).

[20] R. Brand, P. Lunkenheimer, and A. Loidl, J. Chem. Phys. 116, 10386 (2002).

[21] J. K. Krüger, J. Schreiber, R. Jimenez, and K.-P. Bohn, J. Phys.: Condens. Matter 6, 6947 (1994).

[22] U. T. Höchli, K. Knorr, and A. Loidl, Adv. Phys. 39, 405 (1990).

[23] S.-H. Chong, W. Götze, and A. Singh, Phys. Rev. E 63, 011206 (2001).

[24] R. Schilling and T. Scheidsteger, Phys. Rev. E 56, 2932 (1997).

[25] L. Fabbian et al., Phys. Rev. E 60, 5768 (1999).

[26] S.-H. Chong and F. Sciortino, Europhys. Lett. 64, 197 (2003).

[27] S.-H. Chong and F. Sciortino, Phys. Rev. E 69, 051202/1 (2004).

[28] F. Affouard, E. Cochin, R. Decressain, and M. Descamps, Europhys. Lett. 53, 611 (2001).

[29] F. Affouard, A. Hédoux, Y. Guinet, T. Denicourt, and M. Descamps, J. Phys.: Cond. Matter 13, 7237 (2001).

[30] F. Affouard and M. Descamps, Phys. Rev. Lett. 87, 035501 (2001).

[31] M. Bee and J. Amoureux, Mol. Phys. 48, 63 (1983).

[32] M. Foulon, T. Belgrand, C. Gors, and M. More, Acta Cryst. B45, 404 (1989).

[33] B. Kuchta, M. Descamps, and F. Affouard, J. Chem. Phys. 109, 6753 (1998).

[34] F. Affouard and M. Descamps, Phys. Rev. B 59, 9011 (1999).

[35] K. Mirsky and M. D. Cohen, Chemical Physics 28, 193 (1978).

[36] D. C. D, F. Sokolic, M. Descamps, and A. Perera, Mol. Phys. 96, 1033 (1999).

[37] S. Melchionna, G. Ciccotti, and B. L. Holian, Molec. Phys 78, 533 (1993).

[38] K. Kobashi, T. Kyomen, and M. Oguni, J. Phys. Chem Solids 59, 667 (1998).

[39] M. Ricker, F. Affouard, R. Schilling, and M. Descamps, to appear in J. of Non-Cryst. Solids 2006.

[40] E. F. Westrum, in Molecular Dynamics and Structure of Solids, R. S. Carter and J. J. Rush, Eds.; National Bureau of Standards: Washington, 1969.

[41] J. H. Strange, Acta Cryst. B28, 1645 (1972).

[42] J. Min, J. Benet-Buchholz, and R. Goese, Chem. Commun. , 2751 (1998).

[43] M. Ricco et al., J. Chem. Phys. 86, 4198 (1987).

[44] H. J. C. Berendsen, J. P. M. Postma, W. F. van Gunsteren, A. DiNola, and J. R. Haak, J. CHem. Phys. 81, 3684 (1984).

[45] S. Kämmerer, W. Kob, and R. Schilling, Phys. Rev. E 56, 5450 (1997). 
[47] S.-H. Chong and W. Götze, Phys. Rev. E 65, 041503/1 (2002).

[48] K. Kishimoto, H. Suga, and S. Seki, Bull. Chem. Soc. Japan 51, 1691 (1978).

[49] H. Stokes, T. Case, D. Ailion, and C. Wang, J. Chem. Phys. 70, 3563 (1979).

[50] L. J. Lewis and G. Wahnström, Phys. Rev. E 50, 3865 (1994).

[51] P. Bordat, F. Affouard, M. Descamps, and F. Müller-Plathe, J. Phys.: Condens. Matter 15, 5397 (2003).

[52] P. Sindzingre and M. L. Klein, J. Chem. Phys. 96, 4681 (1991).

[53] J. H. Crowe, S. B. Leslie, and L. M. Crowe, Cryobiology 31, 355 (1994).

[54] J. L. Green and C. A. Angell, J. Phys. Chem. 93, 2880 (1989).

[55] C. Branca, S. Magazù, G. Maisano, and P. Migliardo, J. Chem. Phys. 111, 281 (1999).

[56] F. Sussich, S. Bortoluzzi, and A. Cesàro, Thermochim. Acta 391, 137 (2002).

[57] P. Bordat, A. Lerbret, J.-P. Demaret, F. Affouard, and M. Descamps, Europhys. Lett. 65, 41 (2004).

[58] A. Lerbret, P. Bordat, F. Affouard, M. Descamps, and F. Migliardo, J. Phys. Chem. B 109, 11046 (2005).

[59] W. D. Cornall et al., J. Am. Chem. Soc. 117, 5179 (1995).

[60] R. Chelli, P. Procacci, G. Cardini, and S. Califano, Chem. Phys. Phys. Chem. 1, 871 (1999).

[61] J. Blieck, F. Affouard, P. Bordat, A. Lerbret, and M. Descamps, Chem. Phys. 317, 253 (2005).

[62] D. J. Tomlinson, Mol. Phys. 25, 735 (1972).

[63] J. Wuttke and W. P. ans S. Pouget, J. Chem. Phys. 105, 5177 (1996).

[64] R. L. David, CRC Handbook of Chemistry and Physics, CRC Press, Boca Raton, FL, 2005.

[65] D. C. Champeney, R. N. Joarder, and J. C. Dore, Mol. Phys. 58, 337 (1986).

[66] M. Descamps et al., Prog. Theor. Phys. Suppl. 126, 207 (1997).

[67] D. Morineau and C. Alba-Simionesco, J. Chem. Phys. 109, 8494 (1998).

[68] F. Mezei, W. Knaack, and B. Farago, Phys. Rev. Lett. 58, 571 (1987).

[69] S. Adichtchev, T. Blochowicz, C. Tschirwitz, V. N. Novikov, and E. Rössler, Phys. Rev. E 68, 011504 (2003).

[70] A. Tolle, J. Wuttke, H. Schober, G. Randl, and F. Fujara, Eur. Phys. J. B 5, 231 (1998).

[71] M. P. Allen and D. J. Tildesley, editors, Computer Simulation of Liquids, Oxford Science, Oxford, 1987.

[72] W. Kob and H. C. Andersen, Phys. Rev. E 51, 4626 (1995).

[73] W. Kob, C. Donati, S. J. Plimpton, P. H. Poole, and S. C. Glotzer, Phys. Rev. Lett. 79, 2827 (1997).

[74] S. Sastry, Phys. Rev. Lett. 85, 590 (2000).

[75] F. Sciortino, W. Kob, and P. Tartaglia, Phys. Rev. Lett. 83, 3214 (1999).

[76] K. L. Ngai, J. Non-Cryst. Solids 275, 7 (2000).

[77] W. T. Laughlin and D. R. Uhlmann, J. Phys. Chem. 76, 2317 (1972).

[78] C. A. Angell, J. Non-Cryst. Solids 131-133, 13 (1991).

[79] D. J. Plazek and K. L. Ngai, Macromolecules 24, 1222 (1991).

[80] R. Böhmer and C. A. Angell, Phys. Rev. B 45, 10091 (1992).

[81] R. Böhmer, K. L. Ngai, and C. A. Angell, J. Chem. Phys. 99, 4201 (1993).

[82] P. Bordat, F. Affouard, M. Descamps, and K. L. Ngai, Phys. Rev. Lett. 93, 105502 (2004).

[83] T. Scopigno, G. Ruocco, F. Sette, and G. Monaco, Science 302, 849 (2003).

[84] P. Bordat, F. Affouard, M. Descamps, and K. L. Ngai, To appear in J. Non-Cryst. Solids (2006).

[85] R. Chelli, P. Procacci, G. Cardini, and S. Califano, Phys. Chem. Chem. Phys. 1, 879 (1999). 


\begin{tabular}{llcccc} 
Page 13 & Of 30 & & & & \\
& Site-Site & $\mathrm{p}$ & $\mathrm{q}$ & $\epsilon(\mathrm{kJ} / \mathrm{mol})$ & $\sigma(\mathrm{A})$ \\
\cline { 2 - 6 } 1 & $\mathrm{Cl}-\mathrm{Cl}$ & 12 & 6 & 1.441 & 3.350 \\
\cline { 2 - 6 } 2 & $\mathrm{Cl}-\mathrm{Adm}$ & 14 & 8 & 3.087 & 4.786 \\
\cline { 2 - 6 } & $\mathrm{Adm}-\mathrm{Adm}$ & 16 & 11 & 12.47 & 6.200 \\
4 & & & & & \\
\cline { 2 - 5 }
\end{tabular}

5

6

7

10

11

12

13

14

15

16

17

18

19

20

21

22

23

24

25

26

27

28

29

30

31

32

33

34

35

36

37

38

39

40

41

42

43

44

45

46

47

48

49

50

51

52

53

54

55

56

57

58

59

60

http://mc.manuscriptcentral.com/tandf/jenmol 


\begin{tabular}{cccc} 
Site-Site & $\mathrm{A}\left(\mathrm{kJ} / \mathrm{molA}^{6}\right)$ & $\mathrm{B}(\mathrm{kJ} / \mathrm{mol})$ & $\mathrm{C}\left(\AA^{-1}\right)$ \\
\hline $\mathrm{C}-\mathrm{C}$ & 2378.1 & 350142 & 3.60 \\
\hline $\mathrm{C}-\mathrm{H}$ & 523.1 & 36701 & 3.67 \\
\hline $\mathrm{H}-\mathrm{H}$ & 114.3 & 11112 & 3.74 \\
\hline
\end{tabular}

5

6

7

8

9

10

11

12

13

14

15

16

17

18

19

20

21

22

23

24

25

26

27

28

29

30

31

32

33

34

35

36

37

38

39

40

41

42

43

44

45

46

47

48

49

50

51

52

53

54

55

56

57

58

59

60

http://mc.manuscriptcentral.com/tandf/jenmol 


\begin{tabular}{llccc}
\multicolumn{4}{l}{ Table 3. Simulation coefficients of DFTCE using a Buckingham potenti } \\
Page 15 & So- & & & \\
& Site-Site & $A(\mathrm{~kJ} / \mathrm{mol})$ & $\rho\left(\mathrm{A}^{-1}\right)$ & $C\left(\mathrm{~kJ} / \mathrm{molA}^{6}\right)$ \\
\cline { 2 - 5 } 1 & $\mathrm{C}-\mathrm{C}$ & 226307 & 0.288 & 2420 \\
2 & $\mathrm{C}-\mathrm{F}$ & 196747 & 0.260 & 1168 \\
3 & $\mathrm{C}-\mathrm{Cl}$ & 390940 & 0.284 & 3864 \\
4 & $\mathrm{~F}-\mathrm{F}$ & 171038 & 0.237 & 565 \\
5 & $\mathrm{~F}-\mathrm{Cl}$ & 320883 & 0.258 & 1808 \\
6 & $\mathrm{Cl}-\mathrm{Cl}$ & 586389 & 0.284 & 5798 \\
7 & & & & \\
\hline
\end{tabular}

8

9

10

11

12

13

14

15

16

17

18

19

20

21

22

23

24

25

26

27

28

29

30

31

32

33

34

35

36

37

38

39

40

41

42

43

44

45

46

47

48

49

50

51

52

53

54

55

56

57

58

59

60

http://mc.manuscriptcentral.com/tandf/jenmol 


\begin{tabular}{lccc} 
Interaction & A-A & A-B & B-B \\
\hline$\varepsilon=\frac{E_{0}}{6_{0}}$ & 1.0 & 0.50 & 1.5 \\
$\sigma=\frac{r_{0}}{2^{1 / 6}}$ & 1.0 & 0.88 & 0.8
\end{tabular}

2

3

4

5

6

7

8

9

10

11

12

13

14

15

16

17

18

19

20

21

22

23

24

25

26

27

28

29

30

31

32

33

34

35

36

37

38

39

40

41

42

43

44

45

46

47

48

49

50

51

52

53

54

55

56

57

58

59

60

http://mc.manuscriptcentral.com/tandf/jenmol 
Figure 1. Reorientational correlation function $C_{2}(t)$ as function of time at different temperatures for a) CLADM, b) NBE and c)
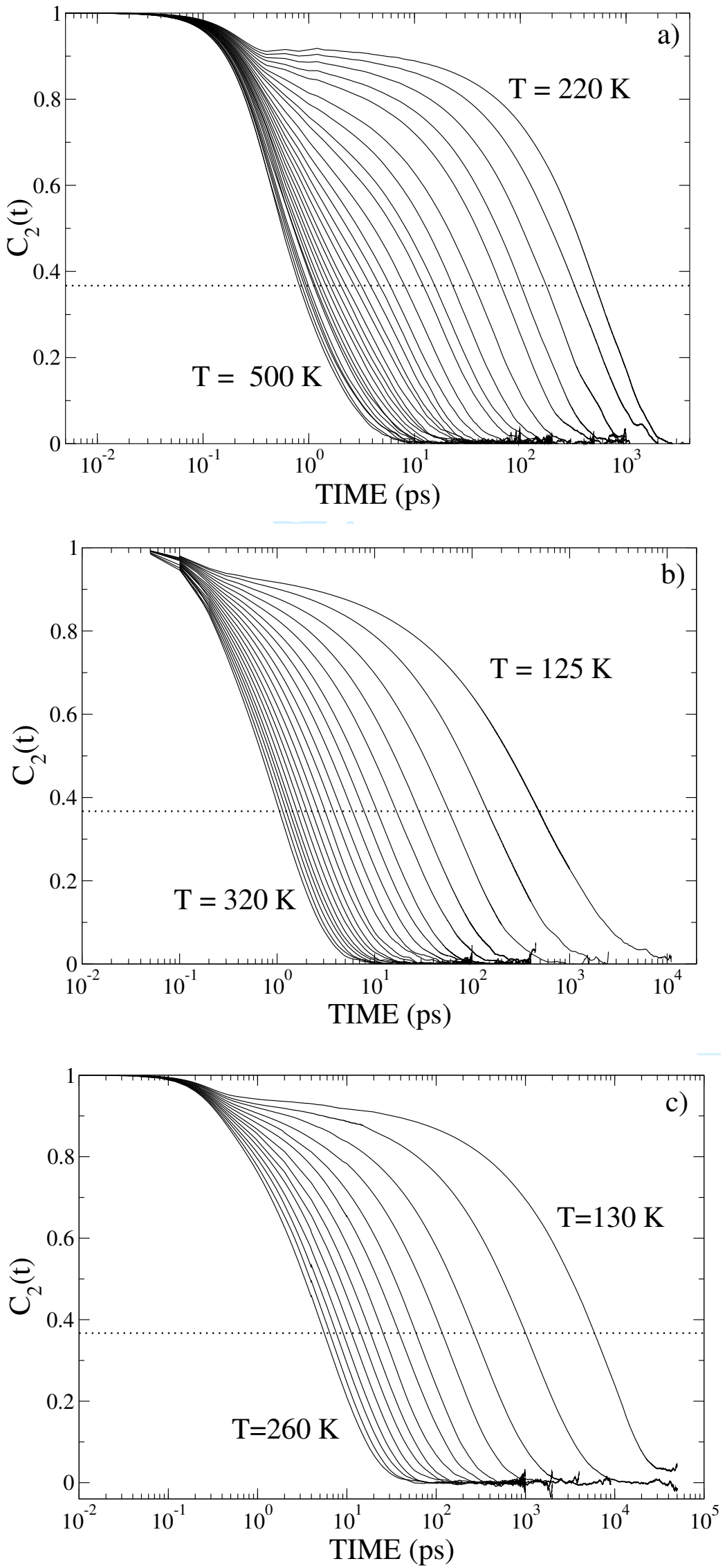

http://mc.manuscriptcentral.com/tandf/jenmol 
Figure 2. Snapshots of the projections of the $\vec{u}$ dipolar moments belonging to one $x y$ crystallographic plane at different instants in a

run of $250 \mathrm{ps}$ at $T=400 \mathrm{~K}$ (top) and $220 \mathrm{~K}$ (bottom). At $T=220 \mathrm{~K}$, Some orientations are not populated since the system is notPage 18 of $\mathbf{3 0}$ completely equilibrated over this duration.
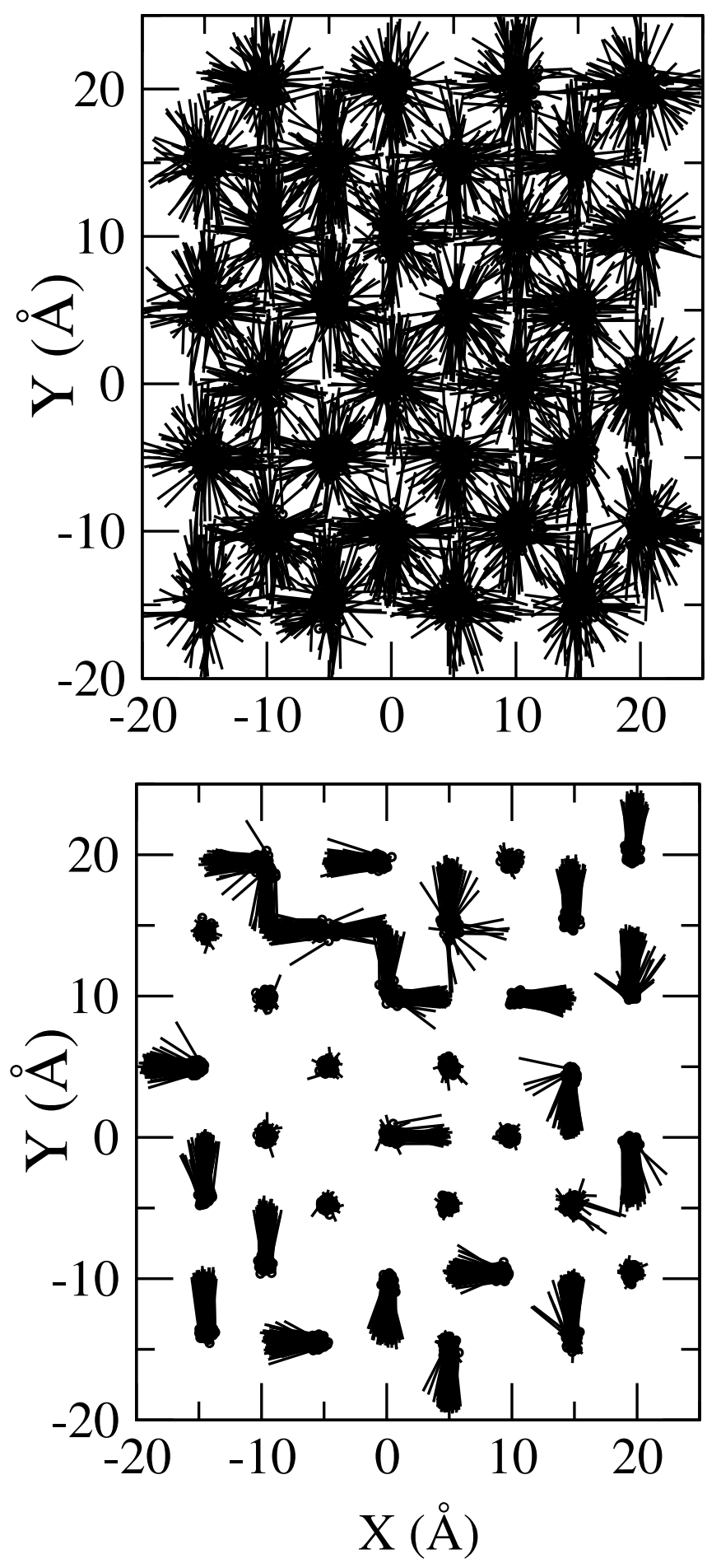

http://mc.manuscriptcentral.com/tandf/jenmol 


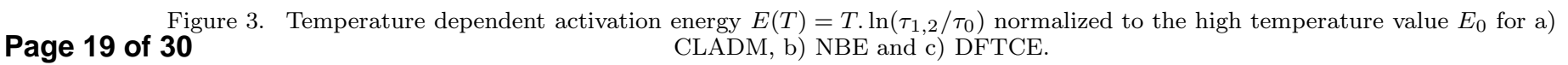
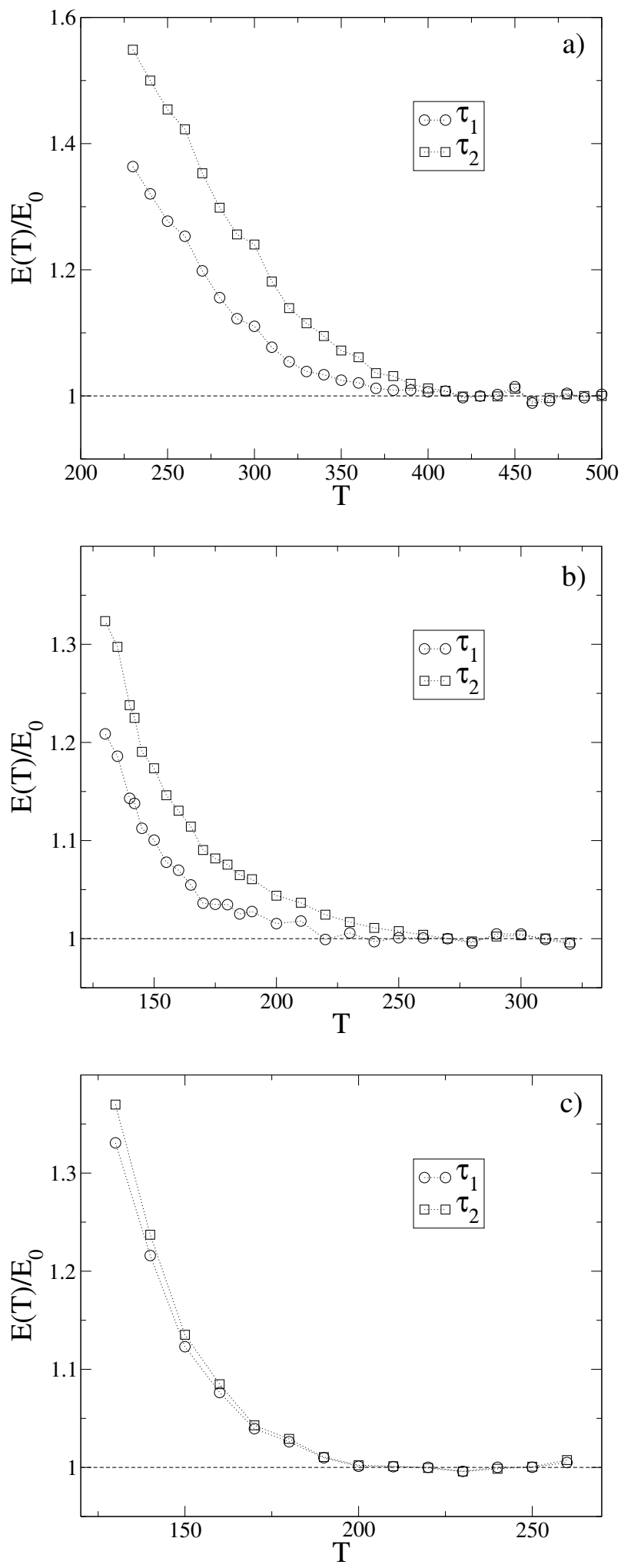

http://mc.manuscriptcentral.com/tandf/jenmol 
Figure 4. The ratio of the relaxational times $\tau_{1} / \tau_{2}$ is displayed as function of the temperature for CLADM, NBE and DFTCE models.

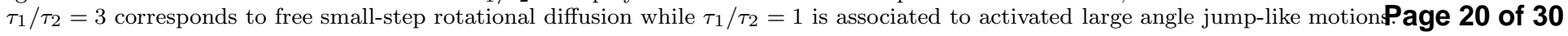

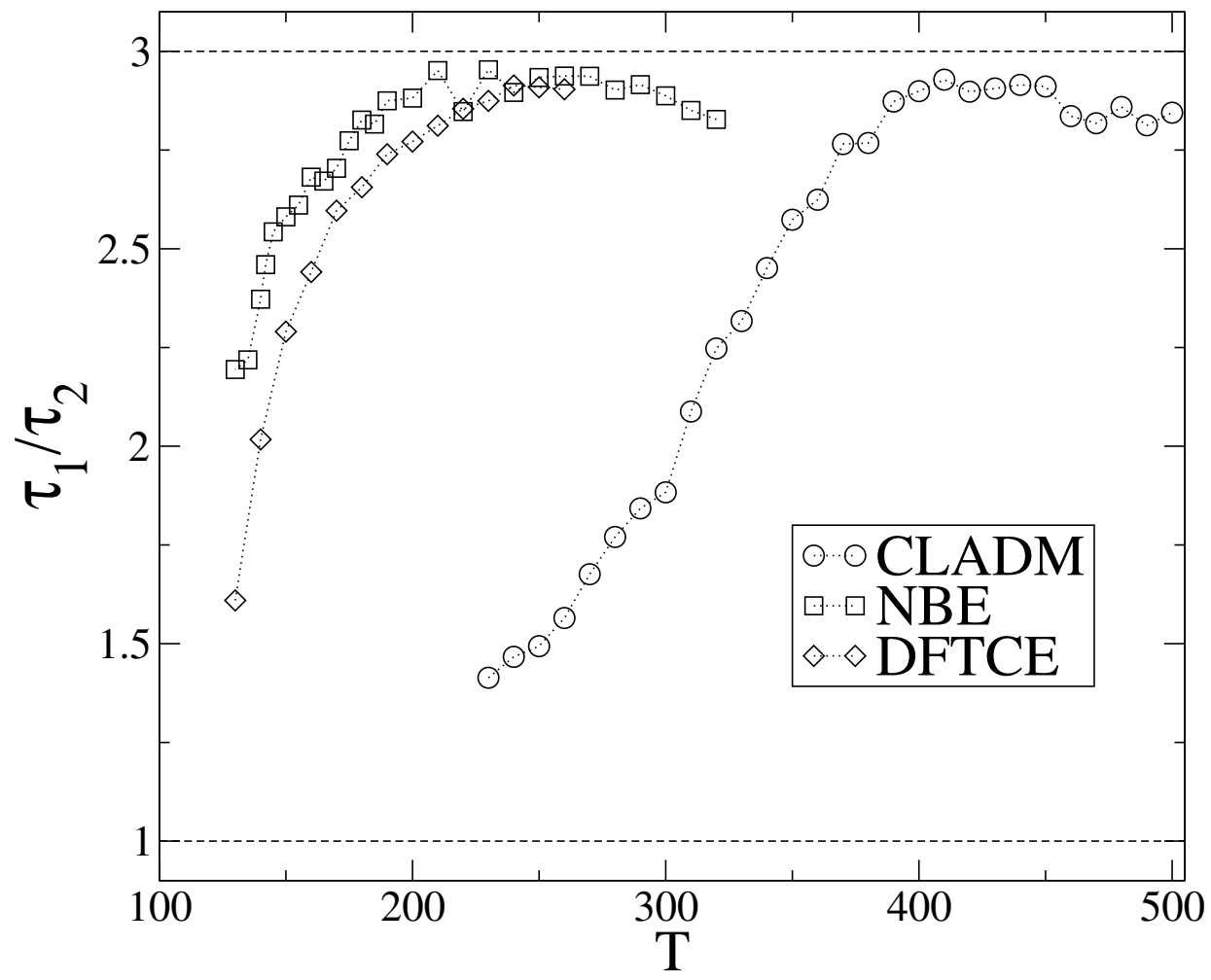



Figure 5. (a) Static structure factor $S(Q)$ obtained from experiments (full circle) at $\mathrm{T}=296 \mathrm{~K}(65)$ and from MD simulations (open
Page 21 of 30 circle) at $\mathrm{T}=333 \mathrm{~K}$. (b) Relaxation times $\tau_{Q} / \tau_{Q_{\max }}$ extracted from $S(Q, t)$ at $\mathrm{T}=333 \mathrm{~K}$, where $\tau_{Q_{\max }}$ is about 148 ps.

1

2

3

4

5

6

7

8

9

10

11

12

13

14

15

16

17

18

19

20

21

22

23

24

25

26

27

28

29

30

31

32

33

34

35

36

37

38

39

40

41

42

43

44

45

46

47

48

49

50

51

52

53

54

55

56

57

58

59

60
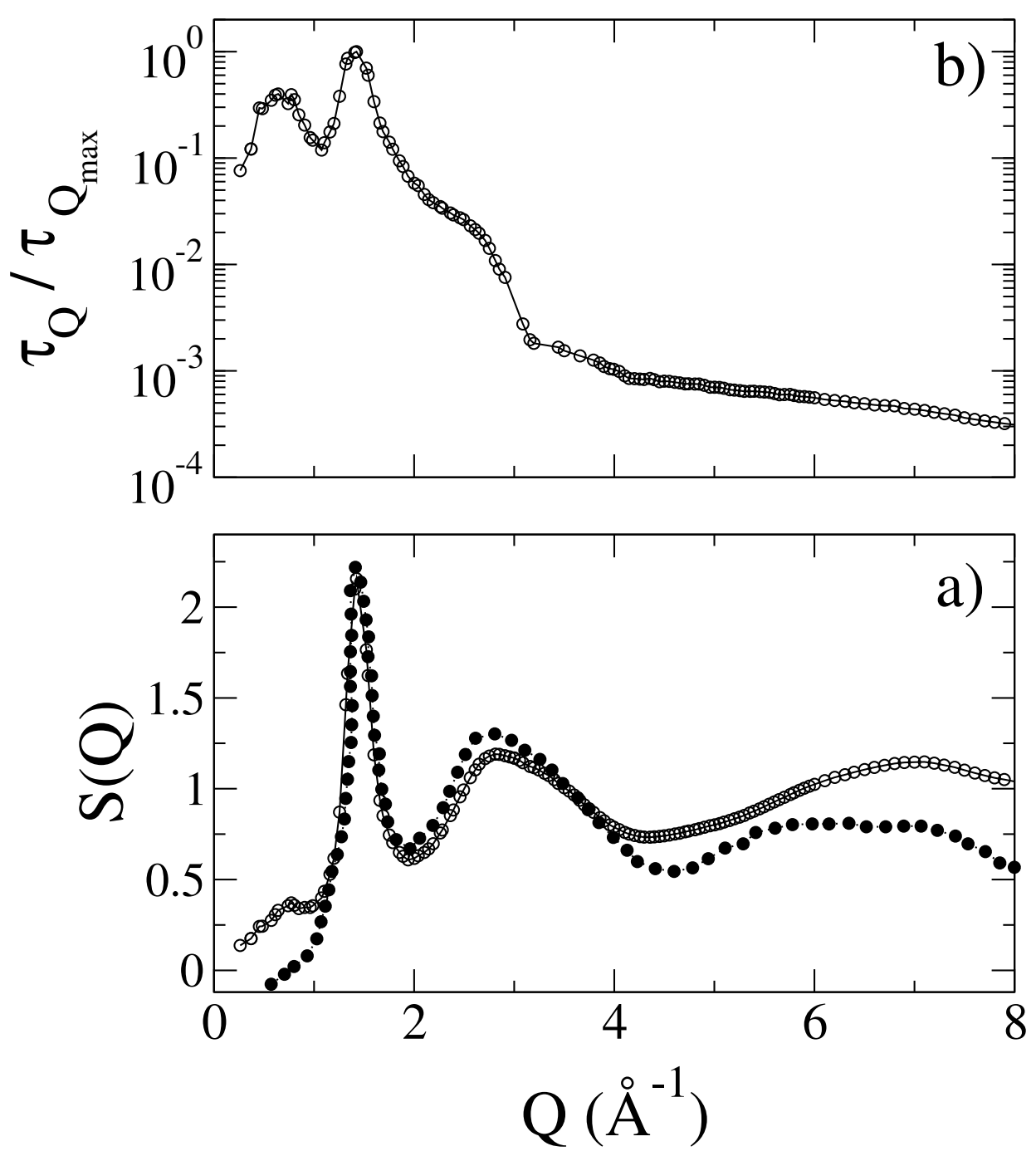

http://mc.manuscriptcentral.com/tandf/jenmol 
Figure 6. Coherent intermediate scattering function $S(Q, t)$ at $\mathrm{Q}=\mathrm{Q}_{\max }$ obtained at $\mathrm{T}=313,333,353,393$ and $413 \mathrm{~K}$ from experiments (full square) and MD simulations (solid line).

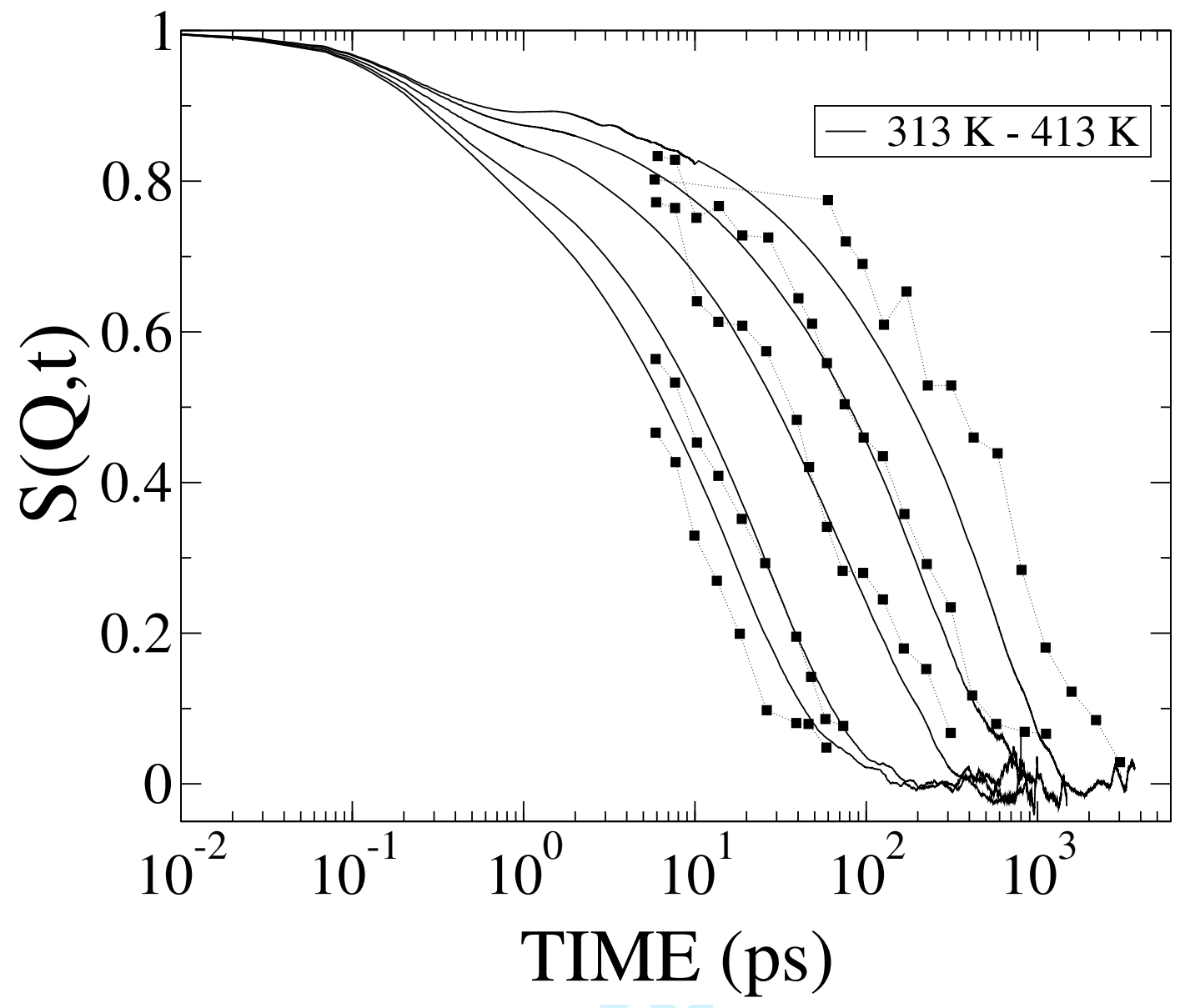




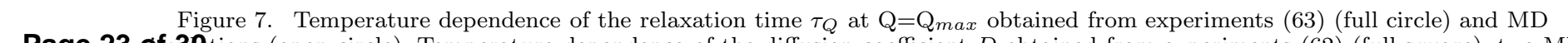

Page 23 ofn3Qtions (open circle). Temperature dependence of the diffusion coefficient $D$ obtained from experiments (62) (full square), two MD simulations (open square (61), open triangle (85)).

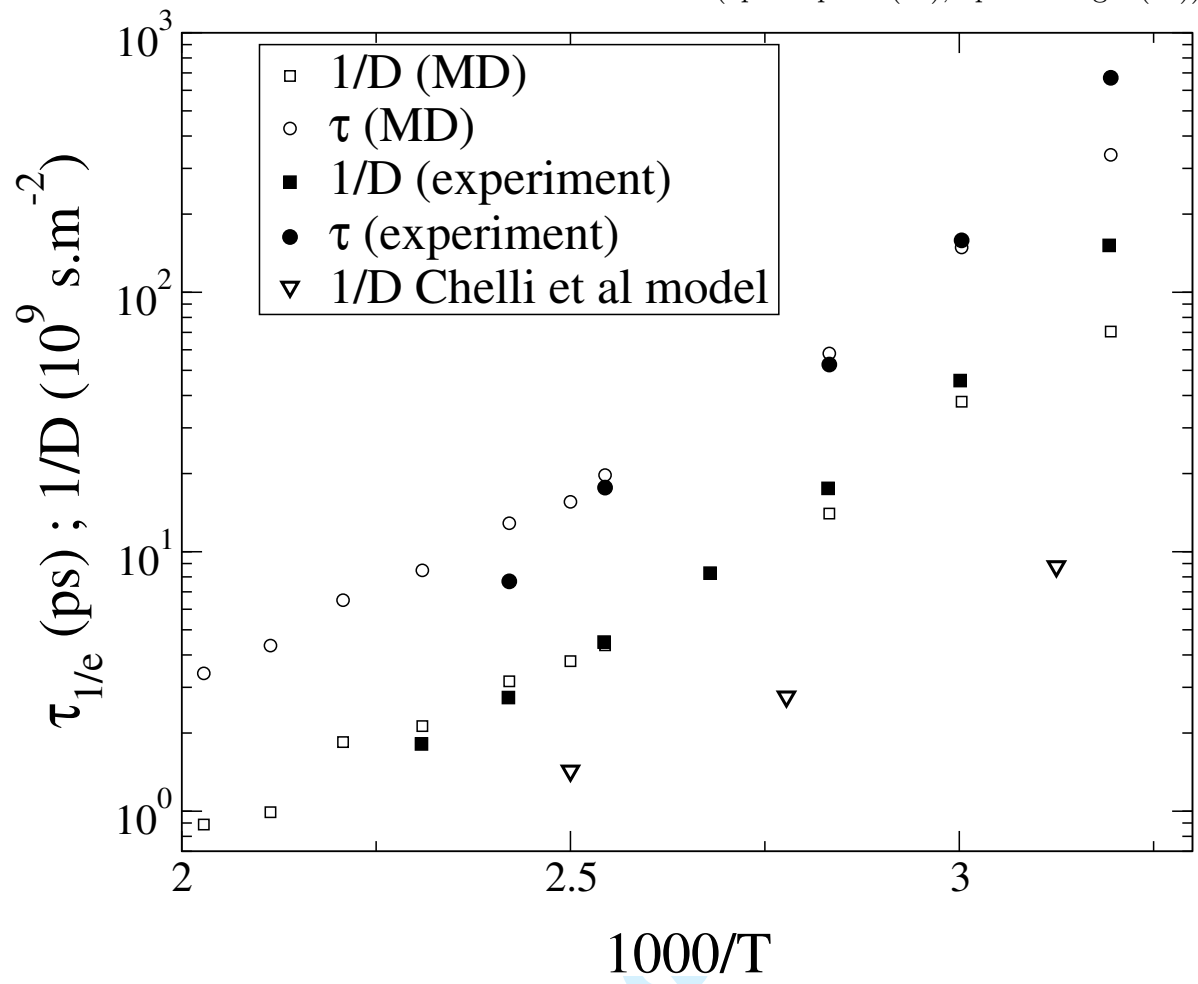


Figure 8. Potential $V(\mathrm{r})$ determining the A-A interaction. The dashed curve is the $(12,11)$ LJ potential for model I, the solid curve is the $(12,6) \mathrm{LJ}$ potential for model II and the dotted curve is the $(8,5)$ LJ potential for model III.

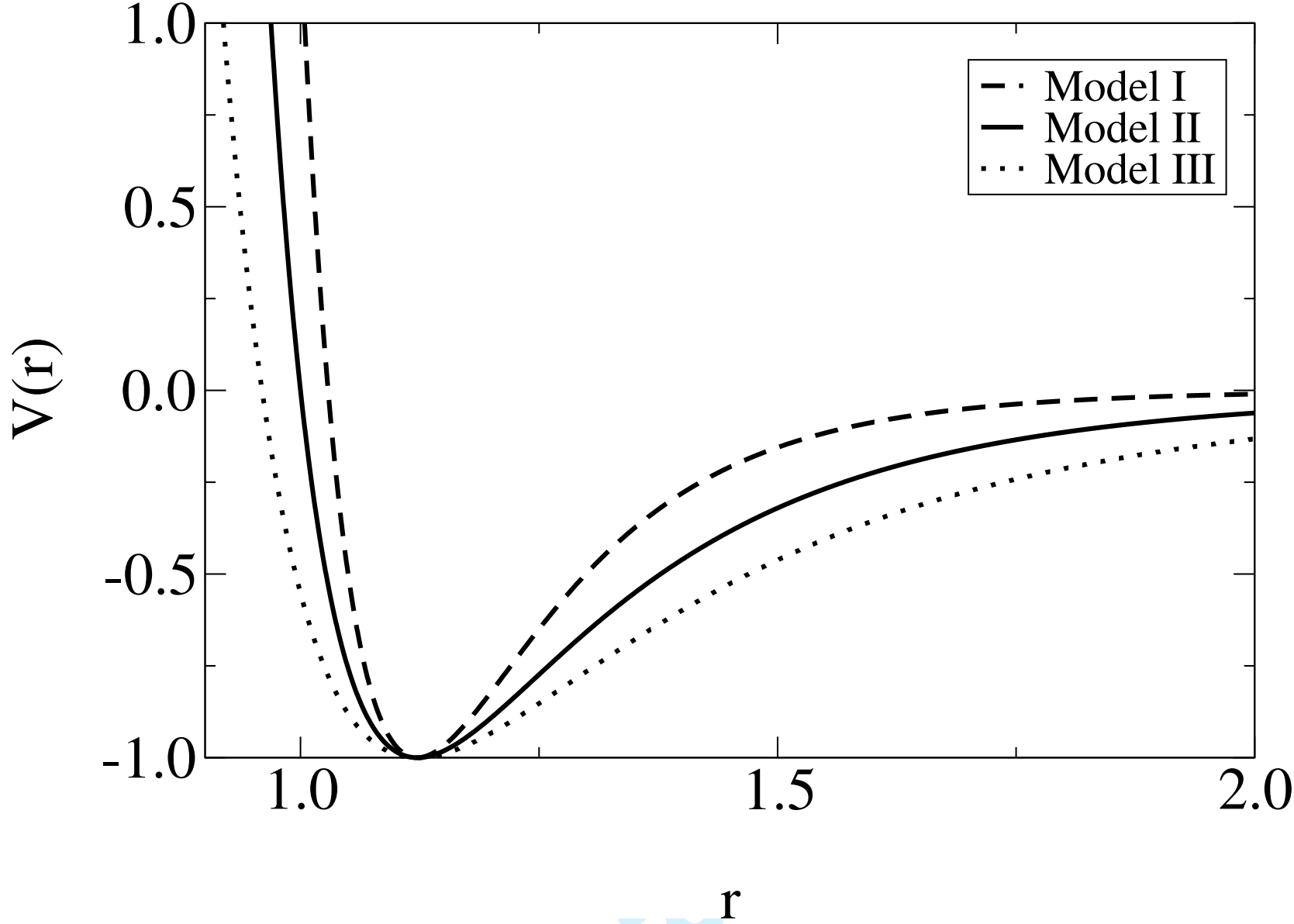

30

31

32

33

34

35

36

37

38

39

40

41

42

43

44

45

46

47

48

49

50

51

52

53

54

55

56

57

58

59

60 
$\begin{array}{cc}\text { Figure } 9 . & \alpha \text { relaxation time } \tau^{-1 / \gamma} \text { of species A versus } T / T_{C} \text {. Data can be fitted with a straight line } \sim\left(T / T_{C}-1\right) \text { for Model I }(\bullet), \\ \text { Page } 25 \text { of } \mathbf{3 0} & \text { Model II }(\mathbf{\bullet}) \text { and Model III }(\mathbf{\square})\end{array}$

1

2

3

4

5

6

7

8

9

10

11

12

13

14

15

16

17

18

19

20

21

22

23

24

25

26

27

28

29

30

31

32

33

34

35

36

37

38

39

40

41

42

43

44

45

46

47

48

49

50

51

52

53

54

55

56

57

58

59

60

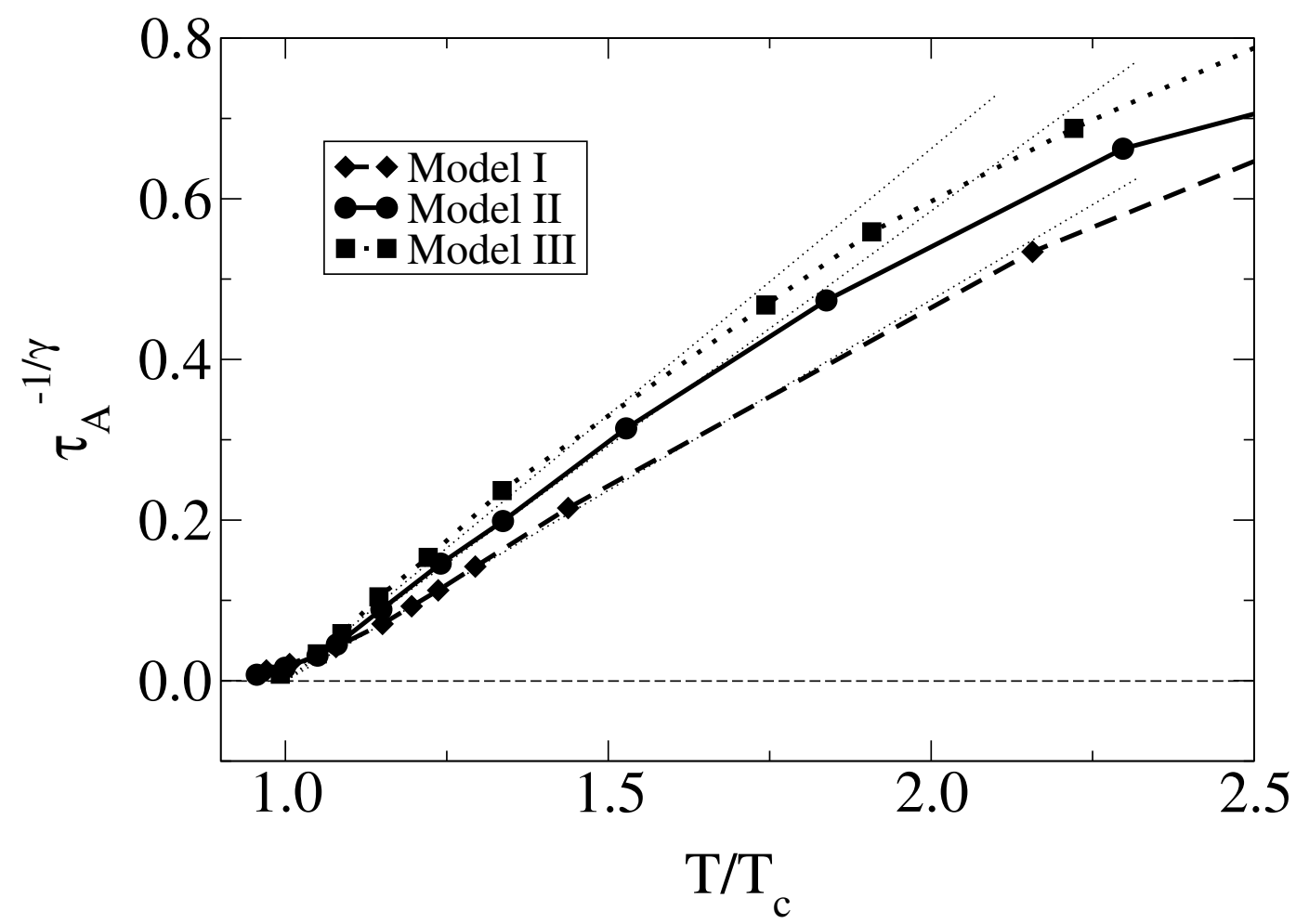

http://mc.manuscriptcentral.com/tandf/jenmol 
Figure 10. Check of the superposition principle of the self intermediate scattering functions $F_{S}\left(Q_{0}, t / \tau_{A}\right)$ for Model III. A detailed view is given in the inset.

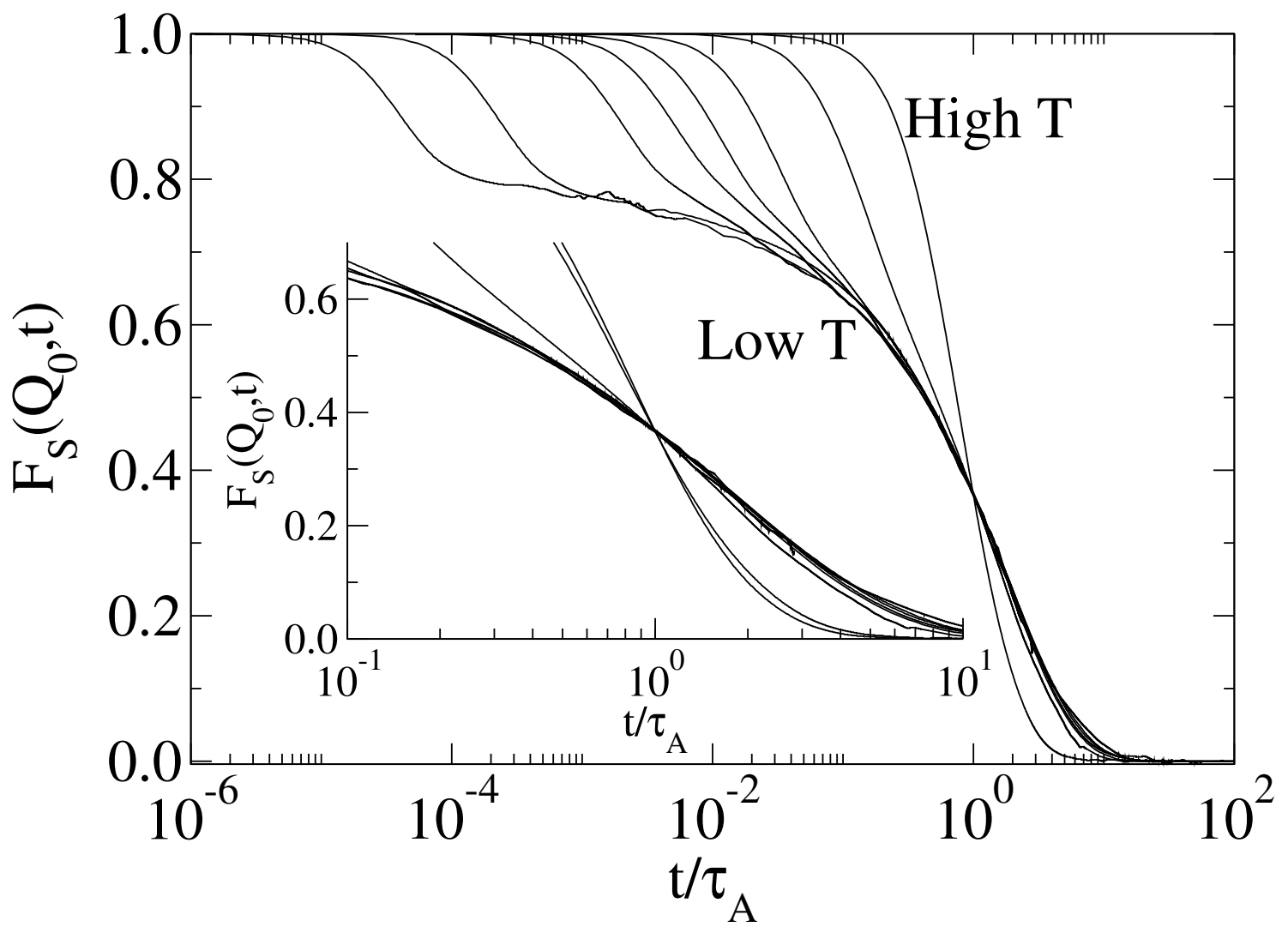


Figure 11. Temperature dependent stretched exponent $\beta$ as a function of $T_{r e f} / T$ for Model I (dashed line), Model II (solid line) and Page 27 of $\mathbf{3 0}$ III (dotted line). $T_{r e f}$ is an arbitrary temperature defined as the temperature for which $\tau_{A}=46435.8$, a very long time $(\approx 10$ ns) compared to the simulation time window.

1
2
3
4
5
6
7
8
9
10
11
12
13
14
15
16
17
18
19
20
21
22
23
24
25
26
27
28
29
30
31
32
33
34
35
36
37
38
39
40
41
42
53
50
50
49
50
50
51
53
54

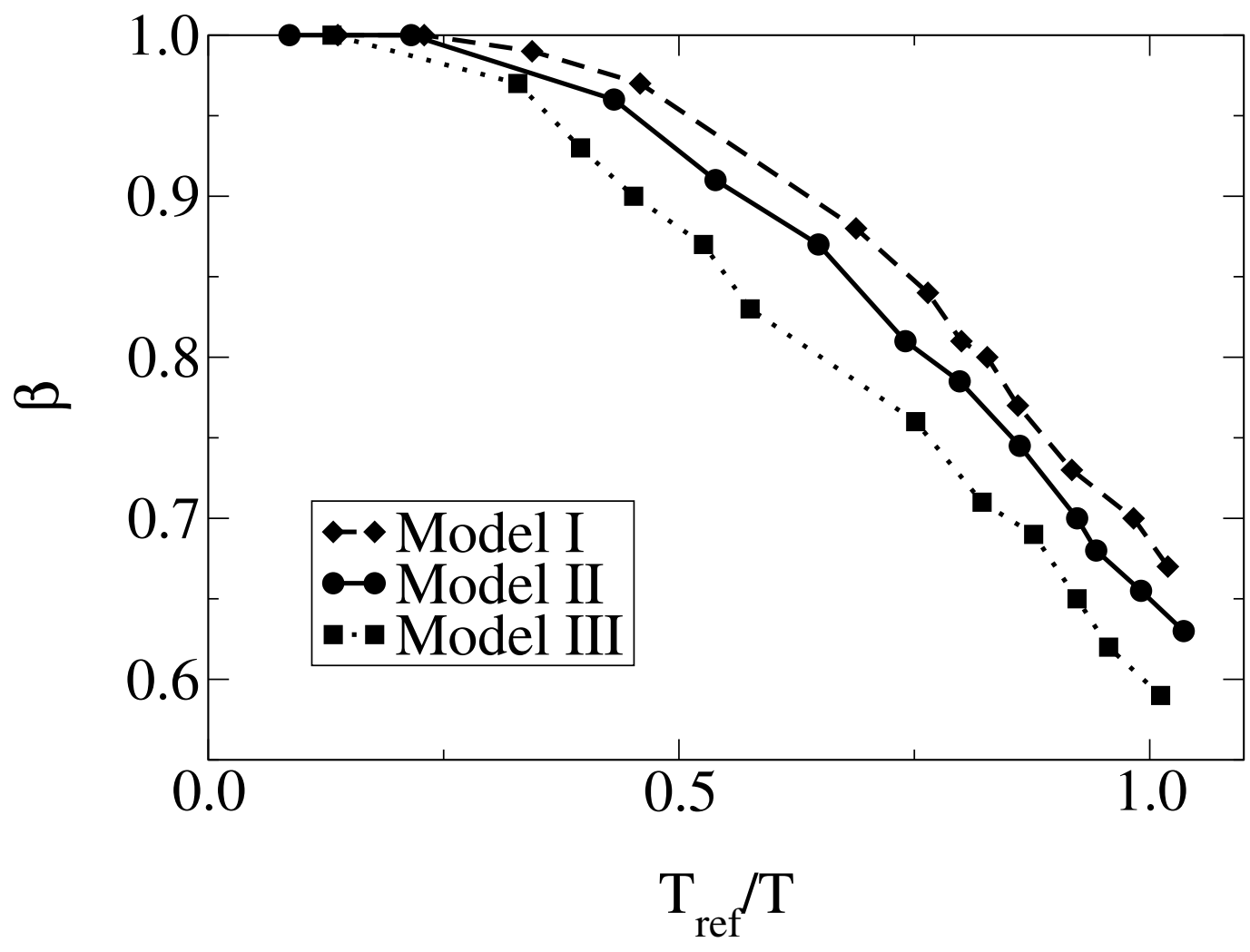


Figure 12. The relaxation times $\tau_{A}$ obtained from $F_{S}\left(Q_{0}, t\right)$ for the three models as a function of $T_{r e f} / T$ where $T_{r e f}$ is defined as the

temperature at which $\tau_{A}$ reaches 46435.8. ( ) Model I, ( ) Model II and (ם) Model III. $T_{r e f}$ is the analogue of $T_{g}$ for simulation Page 28 of 30 when the dynamics of the system slows down to more than $10 \mathrm{~ns}$

1

2

3

4

5

6

7

8

9

10

11

12

13

14

15

16

17

18

19

20

21

22

23

24

25

26

27

28

29

30

31

32

33

34

35

36

37

38

39

40

41

42

43

44

45

46

47

48

49

50

51

52

53

54

55

56

57

58

59

60

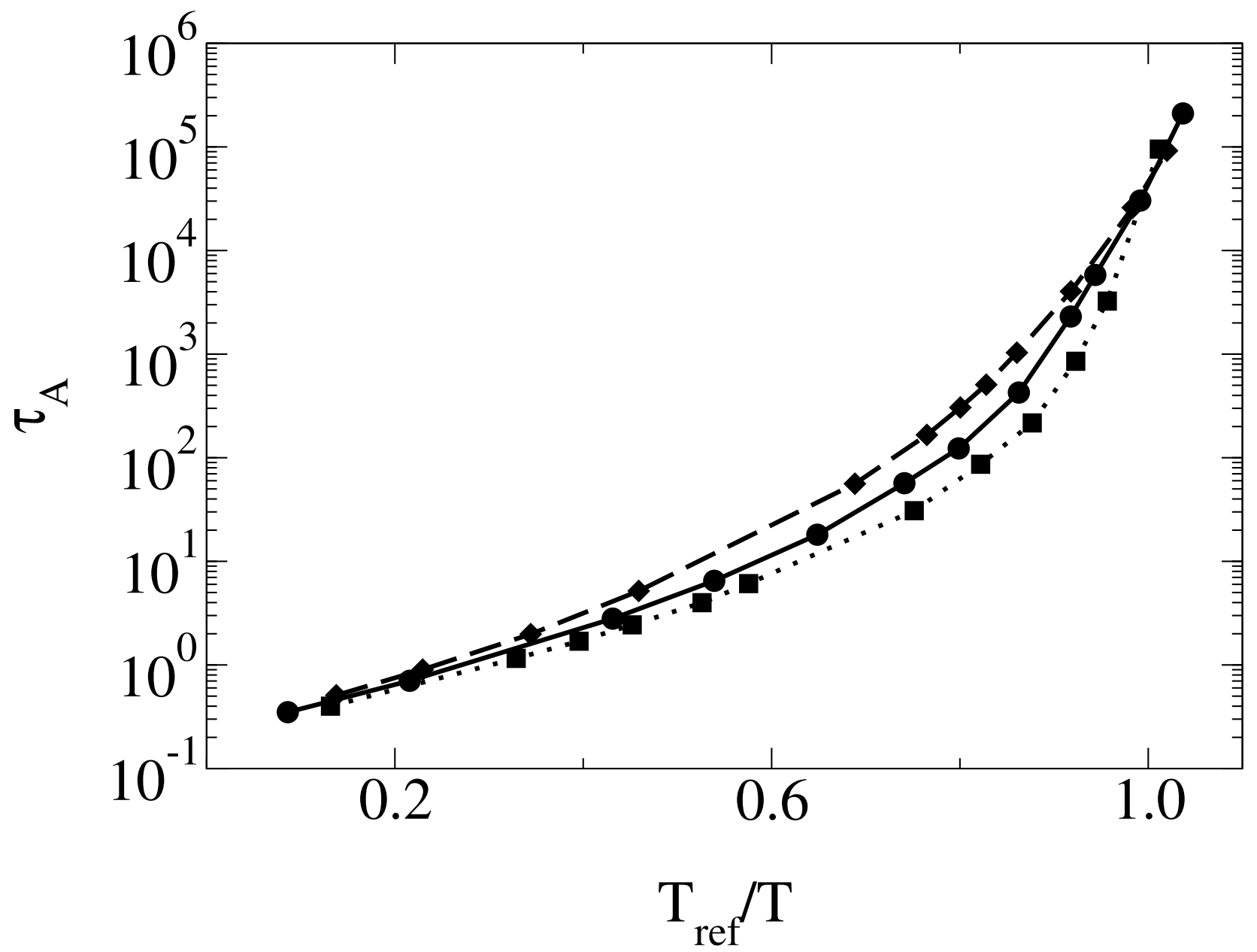




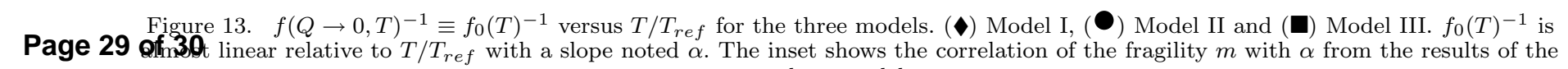
three models.

1
2
3
4
5
6
7
8
9
10
11
12
13
14
15
16
17
18
19
20
21
22
23
24
25
26
27
28
29
30
31
32
33
34
35
36
37
38
39
40
41
42
53
50
50
49
50
50
51
53
54

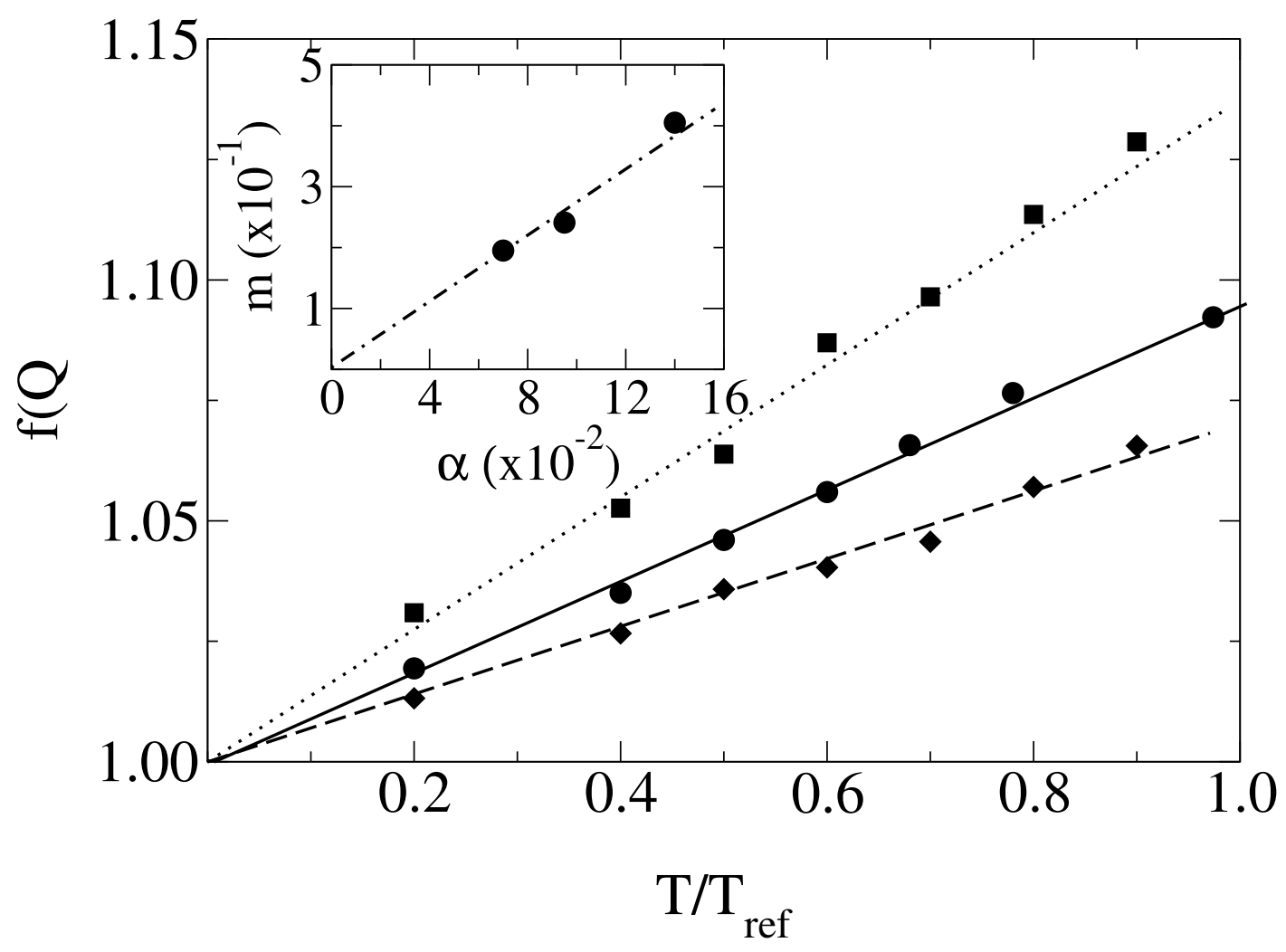

http://mc.manuscriptcentral.com/tandf/jenmol 
Figure 14. Fragilities calculated by different methods for Model I ( $\bullet$, Model II $(\bullet)$ and Model III ( $\mathbf{\square})$. (1) is obtained from Eq. 5 in the text. (2) is the result of $\left.\frac{d \log f}{d T}\right|_{T=T_{r e f}^{-}}$multiplied by 100, where $f$ is the temperature dependent nonergodicity parameter determined at different temperatures below $T_{r e f}$ in the nonequilibrium state identified as a numerical glassy state. (3) is obtained from Sastry's method (2) and multiplied by 20 .

2

5

6

7

8

9

10

11

12

13

14

15

16

17

18

19

20

21

22

23

24

25

26

27

28

29

30

31

32

33

34

35

36

37

38

39

40

41

42

43

44

45

46

47

48

49

50

51

52

53

54

55

56

57

58

59

60

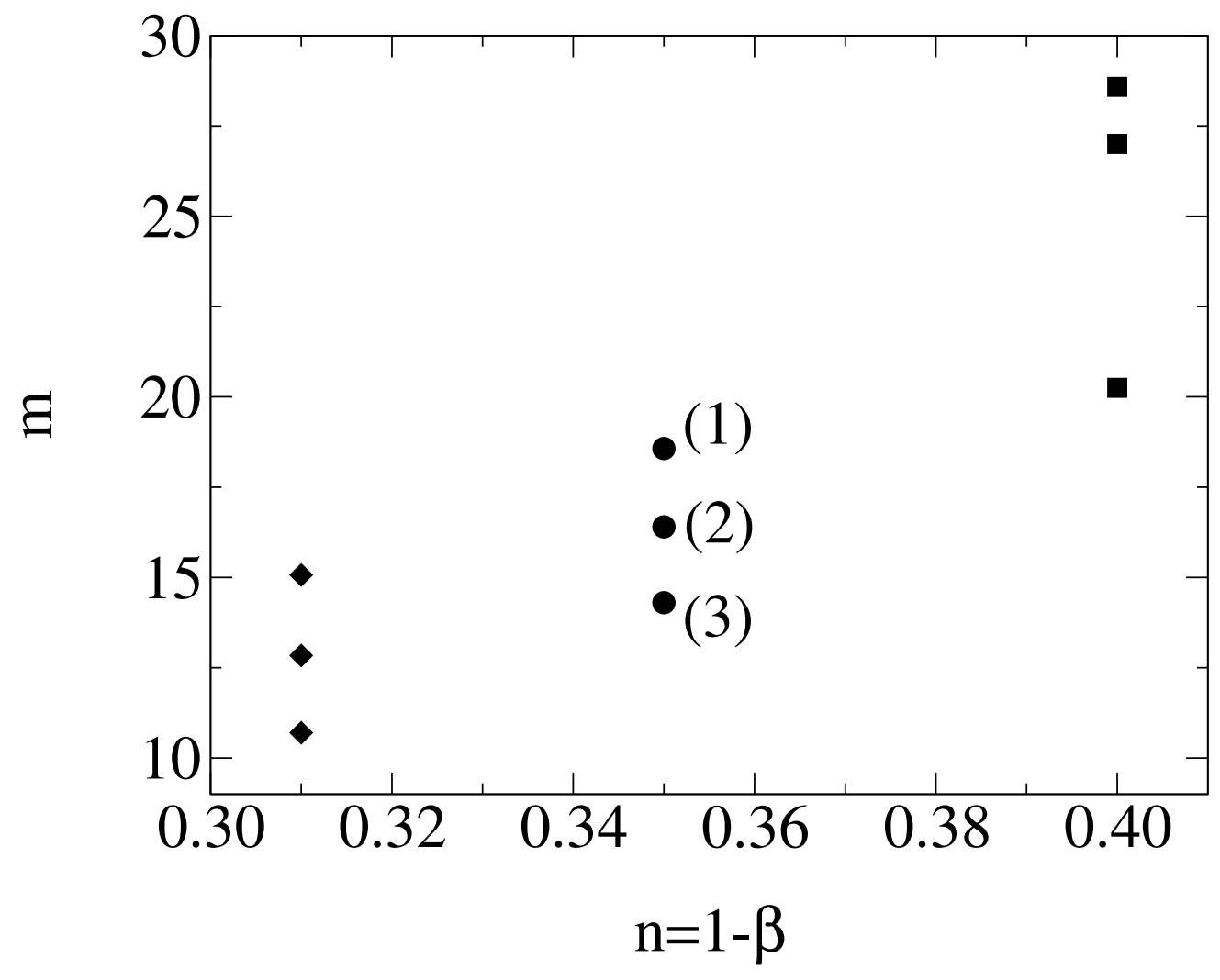

